Research Article

\title{
Study on Cleaning Effect of Different Water Flows on the Pulsed Cavitating Jet Nozzle
}

\author{
Chengting Liu $\mathbb{D}^{1,2}$ Gang Liu $\mathbb{D}^{1,},{ }^{1,2}$ and Zuoxiu Yan $\mathbb{D}^{1,2}$ \\ ${ }^{1}$ School of Petroleum Engineering, Northeast Petroleum University, Daqing 163318, China \\ ${ }^{2}$ Key Laboratory of Enhanced Oil Recovery, Northeast Petroleum University, Ministry of Education, Daqing, China
}

Correspondence should be addressed to Gang Liu; 605521991@qq.com

Received 1 March 2019; Revised 8 May 2019; Accepted 28 May 2019; Published 11 June 2019

Academic Editor: Gilbert R. Gillich

Copyright (C) 2019 Chengting Liu et al. This is an open access article distributed under the Creative Commons Attribution License, which permits unrestricted use, distribution, and reproduction in any medium, provided the original work is properly cited.

The method of cleaning by self-excited pulsed cavitating jet was proposed according to cleaning characteristics and requirements of large storage equipment. This method has many advantages compared with other cleaning methods. In order to achieve the optimum cleaning effects, experimental research on working status of the nozzle at different flow rates was conducted and analysis was carried out from the following four aspects: cavitation morphology, pressure pulse frequency, velocity fluctuation amplitude, and erosion effect. The research results showed that flushing effects in the nozzle without cavitation were far below those with cavitation; when the flow rate increased to over $2.7 \mathrm{~m}^{3} / \mathrm{h}$, cavitation began to appear in the chamber. When $Q=7.2 \mathrm{~m}^{3} / \mathrm{h}$, the velocity fluctuation amplitude was about $17.25 \mathrm{~m}$ and pressure fluctuation occurred for 86 times (maximum) within $1 \mathrm{~s}$. During the experiment on erosion effects, the flow rate had little influence on outside diameter of the erosion circle. The erosion rate increased with the increase of the flow rate, reached the peak value at $Q=7.2 \mathrm{~m}^{3} / \mathrm{h}$, but slightly decreased subsequently.

\section{Introduction}

Advantages of the self-excited pulse generation mode include little wear and tear, low energy consumption, simple structure, and extensive application. It has been widely used in cleaning, drilling, rock breaking, etc. [1]. Oil storage tanks and oil pipelines are essential for oil storage and transportation. In the long-time storage and transportation, temperature variation, impurities in oil, environmental factors, and so on will lead to corrosion and rust of oil tanks and pipelines. It will lower the quality of stored and transported oil, reduce production efficiency, and bring terrible safety hazards [2]. To guarantee oil product quality and facilities safety, cleaning and rust removal shall be conducted regularly on oil tanks and pipelines. Cavitating water jet can make bubbles burst and bring extremely high impact pressure and stress concentration in the local area on the surface of an object. It will soon destruct the object surface, thus to clean, crush, and even cut materials.

In cleaning and dust removal process of oil tanks and pipelines, cavitating water jet technology can both improve working performance and guarantee the high quality of cleaning and dust removal. Therefore, it is necessary to study low-pressure and high-flow jet cleaning technology. Advantages of pulsed jet and cavitating jet are combined to propose low-pressure self-excited pulse cavitating jet cleaning devices according to cleaning features and requirements of large storage facilities. The relationship between the geometrical shape of nozzle and the pulse and cavitation effect of water jet is not well understood. It is of great significance to study the relationship between the optimal operating parameters of nozzle and the pulse and cavitation effect by experimentally testing the pressure pulse times of jet flow in the nozzle, studying the pressure distribution of jet flow in the nozzle, and exploring appropriate methods to study the cleaning effect of the nozzle.

Self-excited oscillation pulsed jet is a new high-efficiency jet technology developed based on transient flow theory, hydroacoustic principle, fluid resonance, etc. After extensive research on self-resonating cavitating jet, Johnson and Conn [3] of Tracor Fluid Systems in the United States found two types of most commonly used nozzles for producing self- 
resonating cavitating jet, namely, Helmholtz oscillating nozzle and organ-pipe nozzle, which could generate cavitation phenomenon and quite violent cavitation in the hole with depth 2-6 times that of the ordinary cavitation nozzle. They established a water-jet cavitation device with central body so that the target object could be effectively hit through the cavitation generated in the vortex area formed when jet flow passed through the central body and the repeated adjustment of target distance.

Kubota et al. [4] conducted experimental research on the oscillation system composed of jet flow and wall surface and mixed layer and wall surface. Also, they found the variation relationship among oscillation frequency, cavity length, and jet velocity.

Geveci et al. [5] discussed the influence of collision wall on the characteristics of shear layer based on experimental study, and the research results showed that the structure of shear layer would change when collision wall existed. The existence of collision wall could make the newly generated disturbance feedback return to the shear layer separation area, thus causing the variation in shear layer structure, which could promote the generation of continuous large vortex structure.

Vijay et al. [6] carried out a systematic theoretical and experimental research on the self-oscillating cavitation water jet in the water jet laboratory. The test results showed that, under the same pump pressure, the rock breaking effect of self-oscillating cavitation jet was 2-4 times that of the jet flow with a common conical nozzle and the impact pressure pulsation amplitude was $24 \%$ to $37 \%$ higher than that of common jet flow. Moreover, there was optimal jetting spacing for the rock eroded by self-oscillating cavitation water jet, generally 8-12 times of the nozzle outlet diameter.

Wang and Shen [7] theoretically analyzed Helmholtz oscillation nozzle and deduced the mathematical model. Helmholtz oscillation nozzle was proved to be able to produce enormous pressure oscillation by the erosion test upon standard rock sample.

Deng et al. [8] established a similar network model for the self-oscillating pulsed jet device according to fluid network theory and carried out a numerical analysis on the frequency characteristics of the system. The results showed that the inherent frequency of self-excited oscillation pulsed jet device was mainly determined by nozzle configuration, nozzle structure size, and wave velocity, while the frequency characteristics of the system were influenced by the fluid pressure, nozzle cavity length, etc. And, the range of cavity length for maximum resonance peak was given through calculation in combination with experiment.

Ruliang [9], Jiang et al., [10] and Li et al. [11] studied the mechanism of jet flow through numerical analysis and experimental verification. They believed that the structure and operating parameters of self-excited pulsed cavitating jet device had significant influence on the pulse performance. Gao et al. [12] conducted many numerical simulations and experiments on high-pressure and low-velocity ejectors. Chen et al. [13] and Qu et al. [14, 15] studied the evolution process of low-pressure and high-velocity oscillating cavity.
Powell [16] theoretically analyzed the feedback mechanism of water sound waves and found that the instability characteristic of jet flow mainly depended on Reynolds number, Strouhal number, and orifice edge distance. Many years later, Wilson et al. [17] established the Helmholtz nozzle model and demonstrated that the reason for the excitation of nozzle pulse was the instability of vortex ring formed by jet flow and the interaction between vortex ring and rigid boundary. Crow and Champagne [18] conducted an experimental study on the influence factors of nozzle surface roughness and feed pipe diameter. Howe [19] proposed a theory of Helmholtz nozzle excitation by external perturbation, involving the derivation of Green's function. He claimed that the large-scale structure in the jet shear layer might be caused by the instability of the Kelvin-Helmholtz model.

Ma et al. [20] studied Helmholtz nozzle experimentally and theoretically and proposed a model for predicting pressure fluctuations based on the thickness of approaching boundary layer. Corá et al. [21] provided a new method to design Helmholtz nozzle and theoretically determine its acoustic performance. $\mathrm{Hu}$ et al. [22] put forward a new method for generating pulsed air-water jets. In pulsed water jet study, the experimental equipment of pulsed jet dust collector was designed. When the cylindrical cone is installed inside, the internal pressure and dust collection efficiency can be improved. Tang et al. [23] conducted a study and found that there was an optimal cavity length corresponding to the dynamic jetting pressure.

This paper studied the working parameters of a new jet cavitation nozzle to experimentally study and verify the cavitation effect under different working parameters from 4 aspects, i.e., cavitation morphology, number of pressure pulsations, velocity pulsation amplitude, and erosion test, so as to serve future engineering practice.

\section{Materials and Methods}

2.1. Experimental Equipment and Measurement Methods. The experimental procedures are as shown in Figure 1. The experimental equipment is divided into 2 systems. The first is the hydraulic system: the screw pump will pump the water in the water tank to the pipeline where valves are provided. The flow rate through the nozzle can be adjusted by adjusting the valve opening and pump speed. The flow rate is measured and sent to the computer system by an electromagnetic flowmeter (accuracy class $\pm 0.5 \% \mathrm{R}$ ). Pressure transmitters (RS-845 and HSTL-802 with a sampling frequency of $1,000 \mathrm{~Hz}$ ) are provided in the upstream of the nozzle line and above the nozzle chamber. The second is the image acquisition system composed of laser controller, high-speed camera, and computer. Figure 1 is the flow chart for experimental equipment. This experiment uses screw pump (model: G 40-1 screw pump), which provides high pressure (the maximum 1.2 MPa), high flow $(200 \mathrm{~L} / \mathrm{min})$ to meet experimental requirements. During the experiment, the laser is used as a light sheet. The laser and the high-speed camera are provided by Cube World Technology Development Co., Ltd. Experimental photographs are taken with high-speed 

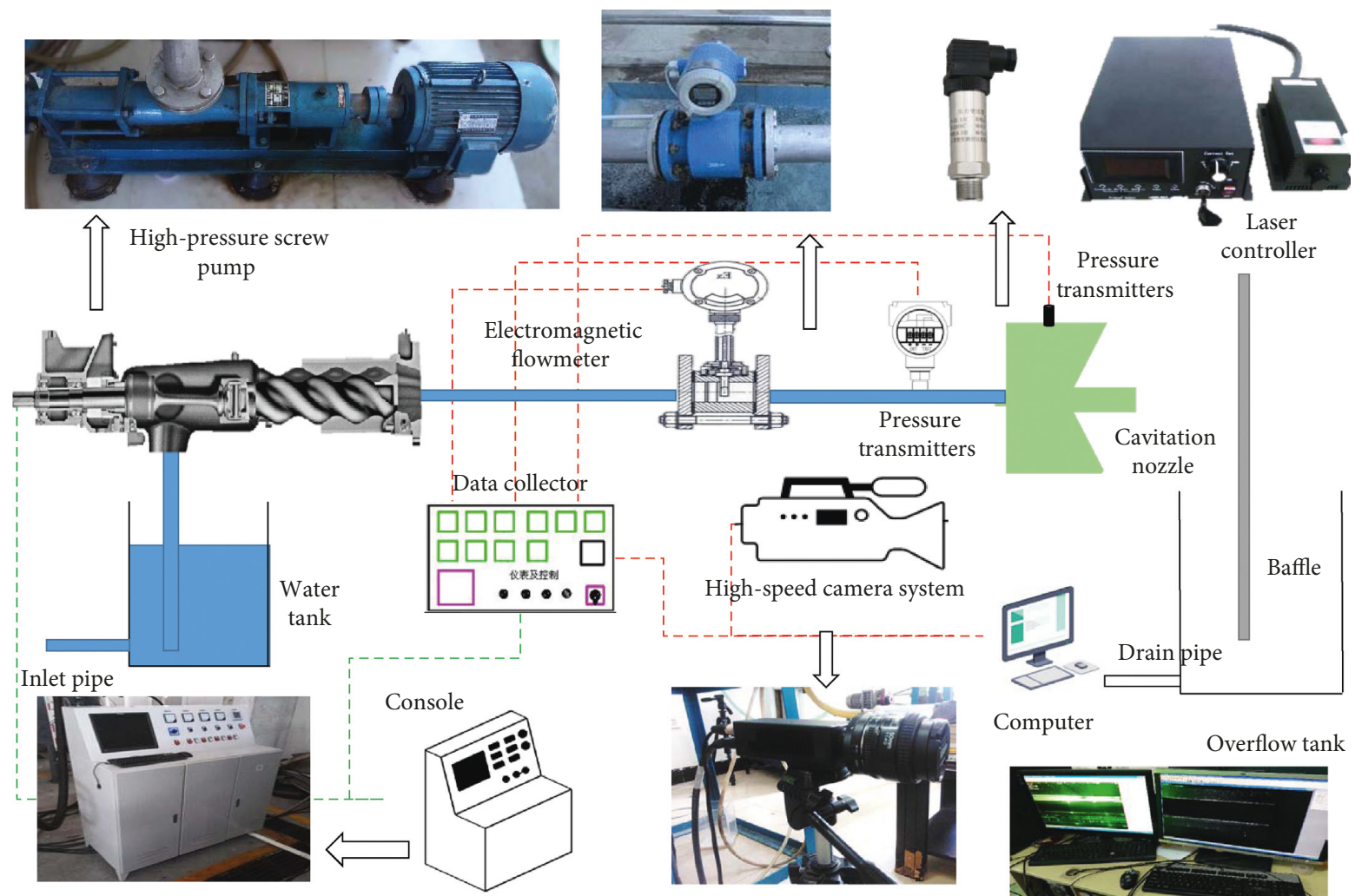

Computer

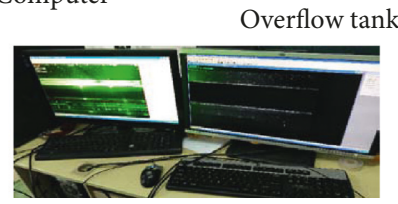

Figure 1: Experimental flow chart.

cameras and digital single lens reflex (DSLR). The model of the DSLR used in the experiment is Nikon D7100. The computer workstation used in the experiment is provided by HP. The adjustable range of the shutter speed of the highspeed camera is $1 / 125 \mathrm{~s} \sim 1 / 12800 \mathrm{~s}$. An image acquisition speed of $60-2500 \mathrm{fps} / \mathrm{s}$ can be realized. The lens of the highspeed camera is Nikon's Sigma 105 mm F2.8 DG Macrolens.

The nozzles used in the experiment are as shown in Figure 2. The diameter of upstream nozzle $D_{\mathrm{u}}=8 \mathrm{~mm}$, chamber diameter $D_{1}=100 \mathrm{~mm}$, the outlet diameter of the downstream nozzle $D_{\mathrm{o}}=16 \mathrm{~mm}$, chamber length $L=60 \mathrm{~mm}$, angle of convergence $54^{\circ}$, the outlet length $L_{\mathrm{o}}=65 \mathrm{~mm}$; the upper opening of the chamber $d=4 \mathrm{~mm}$ is used for connecting pressure sensor.

2.2. Research Methods for Numerical Model. LES separates large-scale eddies and small-scale eddies. Large-scale eddies are directly simulated, while small-scale eddies are enclosed by a model, which can well describe the fluctuation process of the pulsating jet. Therefore, this paper chose LES to simulate the cavitation jet nozzle to solve the instantaneous vortex structure change of self-excited oscillation chamber.

\subsubsection{Impacts of Relative Velocity between Gas Nucleus and} Water. Hsiao et al. [24] derived the cavitation dynamics equation in case of relative velocity between gas nucleus and water and discovered that impacts of this relative velocity $U$ on the expansion and compression of bubbles are equivalent to the equivalent pressure of $\rho U^{2} / 4$ added to the saturated

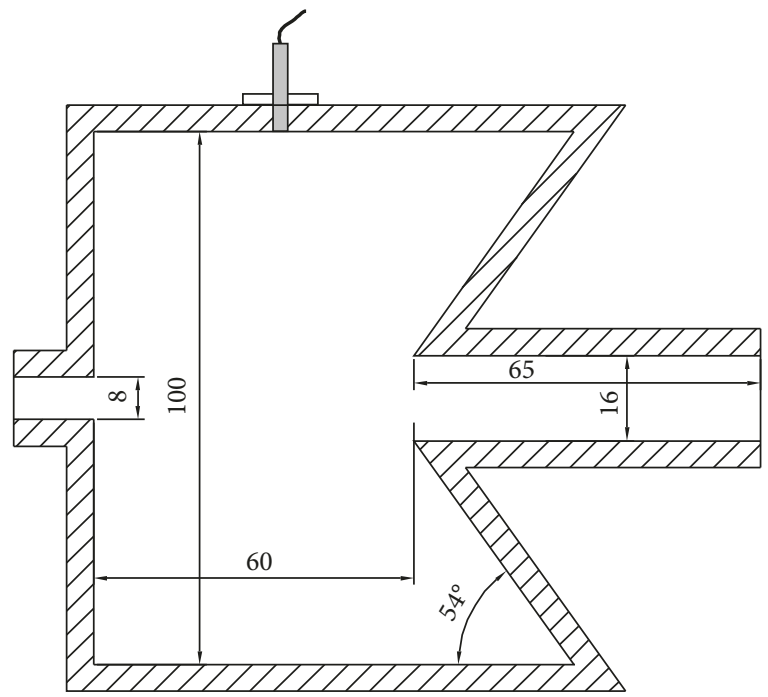

FIgURE 2: Diagram for experimental nozzle.

vapor pressure; thus, the following equation for correcting Rayleigh-Plesset is obtained:

$$
R \frac{D^{2} R}{D t^{2}}+\frac{3}{2}\left(\frac{D R}{D t}\right)^{2}=\frac{P_{\mathrm{R}}-P_{\infty}}{\rho}+\frac{1}{4} U^{2} .
$$

2.2.2. Singhal's Full Cavitation Model. The model first assumes that there is a lot of gas nucleus, the size and quantity of which are fixed values. And, there is no relative velocity 
between gas nucleus and water. The continuity equation for liquid phase is s follows:

$$
\frac{\partial}{\partial t}\left[(1-\alpha) \rho_{\mathrm{l}}\right]+\nabla \cdot\left[(1-\alpha) \rho_{\mathrm{l}} \vec{V}\right]=\left(R_{\mathrm{e}}-R_{\mathrm{c}}\right)
$$

The continuity equation for vapor phase is as follows:

$$
\frac{\partial}{\partial t}\left[\alpha \rho_{\mathrm{v}}\right]+\nabla \cdot\left[\alpha \rho_{\mathrm{v}} \vec{V}\right]=R_{\mathrm{e}}-R_{\mathrm{c}}
$$

The continuity equation for mixed phase is as follows:

$$
\frac{\partial}{\partial t}(\rho)+\nabla \cdot[\rho \vec{V}]=0
$$

Evaporation rate is as follows:

$$
R_{\mathrm{e}} \approx C_{\mathrm{e}} \frac{\sqrt{k}}{\sigma} \rho_{\mathrm{l}} \rho_{\mathrm{v}}\left[\frac{2}{3} \frac{P_{\mathrm{v}}-P}{\rho_{\mathrm{l}}}\right]^{1 / 2}\left(1-f_{\mathrm{v}}-f_{\mathrm{g}}\right) .
$$

Condensation rate is as follows:

$$
R_{\mathrm{c}}=C_{\mathrm{c}} \frac{\sqrt{k}}{\sigma} \rho_{\mathrm{l}} \rho_{\mathrm{v}}\left[\frac{2}{3} \frac{P_{\mathrm{v}}-P}{\rho_{\mathrm{l}}}\right]^{1 / 2} f_{\mathrm{v}} .
$$

Since the impacts of turbulence cannot be neglected, the fluctuation pressure is estimated by Singhal through the following formula in the model:

$$
P_{\text {turb }}^{\prime}=C_{\mathrm{k}} \rho k
$$

where $C_{\mathrm{k}}$ is a constant; the impacts of turbulence on cavitation is finally realized by improving the threshold pressure $P_{\mathrm{v}}$ that controls cavitation, which is obtained by using the following formula:

$$
P_{\mathrm{v}}=P_{\text {sat }}+C_{\mathrm{k}} \rho k
$$

This model needs to solve the above equation and the momentum equation, energy equation, and turbulence model equation for the mixed phase of liquid and vapor. The cavitation model considered the impacts of turbulence and insoluble gas on cavitation and neglected many factors that affect cavitation, such as the relative velocity between gas nucleus and liquid, the viscosity of liquid, surface tension, and velocity gradient. The simulation accuracy of such simplification is acceptable when simulating the steady cavitation problem of fast flow.

2.2.3. Cavitation Number. In the actual measurement, considering the complexity of cavitation and different experimental conditions, usually the cavitation number is used to describe cavitation inception. The cavitation number can be used to judge if cavitation occurs and measure its intensity. When the pressure somewhere in the flow field is less than the saturated vapor pressure of liquids, the cavitation can be shown as follows:

$$
\delta=\frac{2\left(P_{0}-P_{\mathrm{v}}\right)}{\rho V_{0}^{2}} .
$$

Here, $\delta$ is the cavitation number, $P_{\mathrm{v}}$ is the saturated vapor pressure of liquids at a certain temperature, $P_{0}$ and $V_{0}$ refer to reference velocity and reference pressure in the flow field, respectively, and $\rho$ represents liquid density.

2.2.4. Settings of Numerical Simulation. The model adopts $3 \mathrm{D}$ structural mesh to perform numerical calculations. The principal phase is set to water and the second phase vapor; the cavitation model is Schnerr-Sauer; the temperature of the water tank is $26^{\circ} \mathrm{C}$. In such case, the water saturation vapor pressure is $3,362 \mathrm{~Pa}$. The boundary conditions at the inlet select velocity-inlet, with the velocity set to $40 \mathrm{~m} / \mathrm{s}$ (optimum condition); the volume fraction of the incoming water is 1 . The outlet boundary condition was selected as the pressure outlet, and the value was $11325 \mathrm{~Pa}$.

Figure 3 shows schematic diagram of meshing. Meshindependent experiment was conducted. Three groups of mesh with increasing mesh quantity were selected. The impacts of mesh were studied by monitoring the longitudinal velocity distribution of the jet shear layer. $1 \mathrm{~cm}$ longitudinal distance was intercepted at $X=2,4,6$, and $8 \mathrm{~cm}$ on the axis as the monitoring length. The monitoring location is as shown in Figure 4. The model is symmetric, and thus half data were used to draw Figure 5. The monitoring results showed that when mesh $1 X=6 \mathrm{~cm}$, the shear layer has minor displacement. Mesh2 is consistent with mesh3, and thus, mesh 2 was selected as the mesh for numerical simulation. Final information of mesh2: total elements $=1058319$ and total nodes $=1043080$. In addition, under the low-pressure effect of the chamber of cavitation jet nozzle, the shear layer bends and expands to the both sides of the chamber, making the shear layer bend, curl, and form and shed large eddies. Therefore, it is necessary to study the velocity in the neighborhood of the shear layer.

\section{Results and Discussion}

\subsection{Before Cavitation}

3.1.1. Vorticity Partitioning in Cavitation Jet Nozzle. The vorticity can be roughly divided into 7 zones as shown in Figure 6 according to the position where turbulent eddies are generated and the time-averaged distribution: Zone 1 is the high-speed water jet area from the throat pipe; Zone 2 the shear layer of high-speed water jet; Zone 3 the recirculation zone of the outlet nozzle; Zone 4 the zone where centrosymmetric large turbulent eddies are formed; Zones 5 and 6 are the zones where secondary small turbulent eddies caused by large turbulent eddies are generated; Zone 7 is the operation area at the nozzle outlet. High-speed water jet flows into the chamber through the throat (Zone 1) and have shear, disturbance, and mixing with the static water, gradually forming a jet shear layer (Zone 2). Since the jet water is high in speed and forms unstable shear layer and low-pressure zone around the water (as shown in Figure 7). The jet water entrainment and the static water in the shear chamber form centrosymmetric large turbulent eddies (Zone 4). With the continuous formation, rotation, lengthening, and tearing of large turbulent eddies, the processes of which induce the small turbulent eddies in Zones 5 and 6 to generate small eddies. Some of the small 


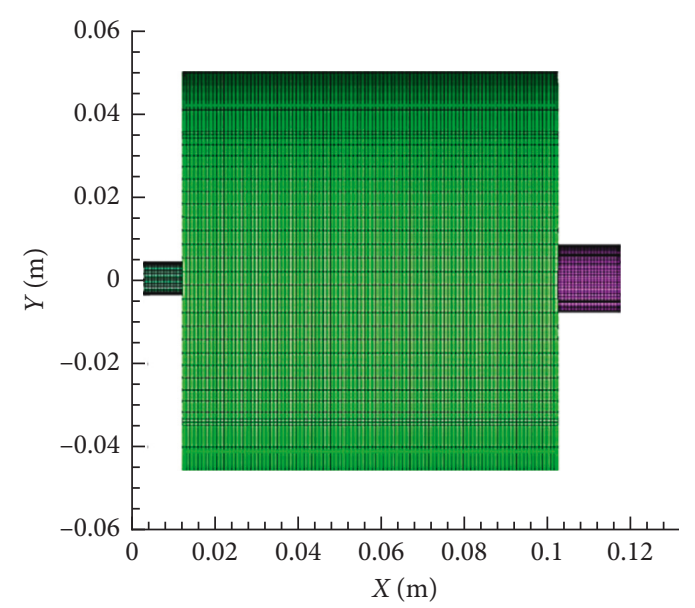

(a)

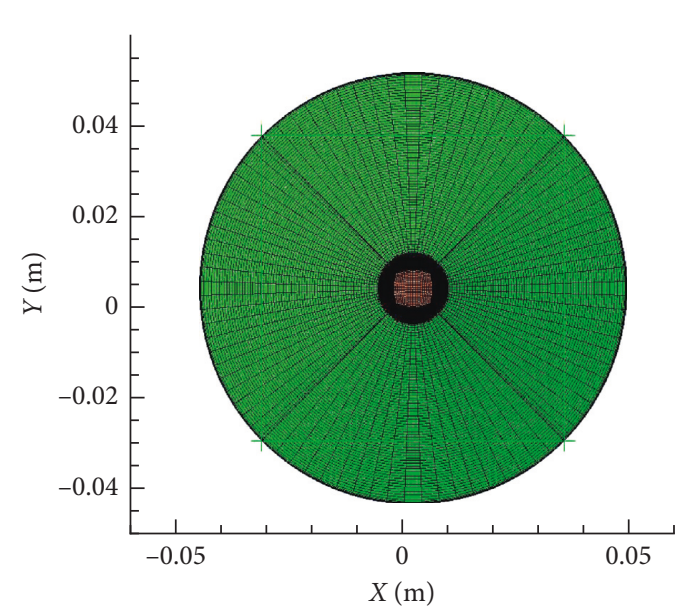

(b)

FIGURE 3: Division diagram for modeling mesh.

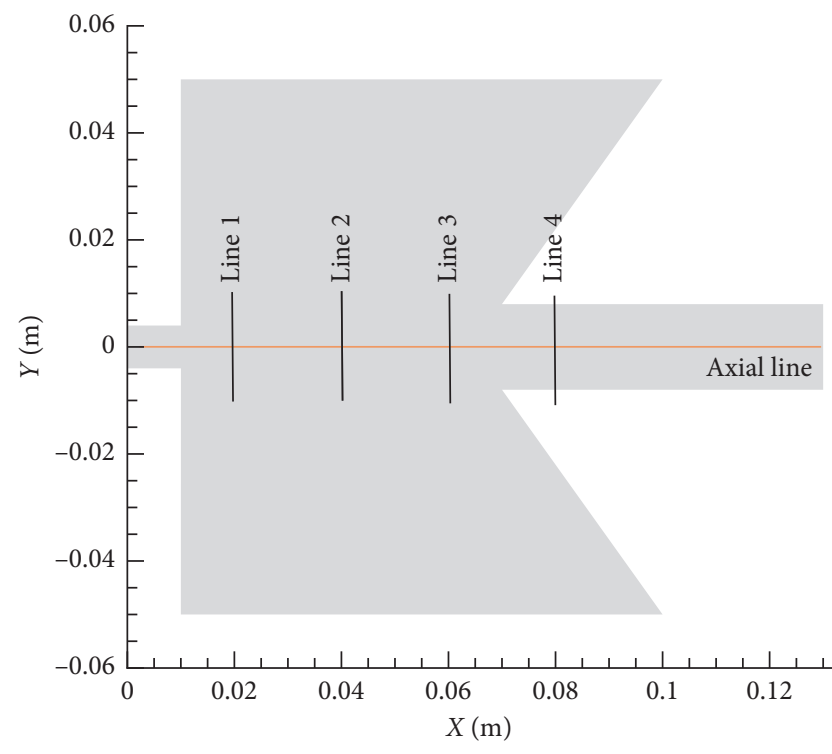

Figure 4: Distribution of monitoring lines.

eddies formed from large turbulent eddies are scattered in the chamber with the rebound on the wall. Thus, a fluctuation cycle is formed in the chamber. Therefore, the periodic fluctuation in the chamber can be regarded as a process where the velocity shear converges to form large eddies, large turbulent eddies are stretched and dissipated in the process of transferring to form new small turbulent eddies, which move along the shear layer, and the energy converges to form large eddies again. The cavitation and turbulent eddy effects of liquid can enhance the instability of the fluid at the nozzle outlet and are featured with periodicity.

As it can be seen from Figure 8(b), the high-speed water jet enters the chamber where it is mixed, forming a turbulent shear layer. There is a pressure gradient around unstable shear layer, forming symmetric discrete large eddies. First of all, crosswise vortexes are formed at the bump of the shear layer. At this time, some energy is stored; thus, it is in an energy storage status. Crosswise vortexes rebound, collide, and move upstream along the shear layer, forming primary spanwise vortexes on Wall 1. Under the action of the pressure gradient, spanwise vortexes leave the shear layer and the vortex structure is lengthened. Vortexes move on Wall 2 and gradually form a large eddy system with a closed loop and then shed to induce new disturbance. In such case, energy is released. Thus, it is in an energy release status. The changes in vortex structure cause different streamlines to come in and out and cause fluctuation effect in the chamber, the increase of vortex scale, and more energy dissipation in the chamber.

3.1.2. Analysis of Cavitation Jet Nozzle Pressure and Velocity Pulsation. The pipeline valve was controlled before cavitation, and the flow rate was increased by further opening the valve. Pressure and flow rate were measured by oscillatory pressure pickup and electromagnetic flowmeter, respectively. It was converted into velocity and the relationship curve for the changes of outlet pressure and velocity in the cavitating jet nozzle over time. Curve data shown in Figure 9 showed that even before cavitation bubbles appeared, pressure and velocity fluctuated in the chamber due to periodic changes of large vortex structure and the fluctuation amplitude would increase with the increase of flow rate. Through comparison, the fluctuation amplitude before cavitation was about $17 \%$ of that after cavitation and was far below that in the chamber during cavitation.

\subsection{Cavitation}

3.2.1. Influence of Turbulence Intensity on Cavitation. Turbulence is currently viewed as a collection of vortices with different scales and intensities. These vortices have great influences on cavitation, and vortex nucleus pressure is very low sometimes, but enough for expansion of gas nucleus. Cavitation possibly occurs in vortices where vortex nucleus pressure is lower than the minimum pressure. It is generally believed that turbulent fluctuation is nonlinear pressure 


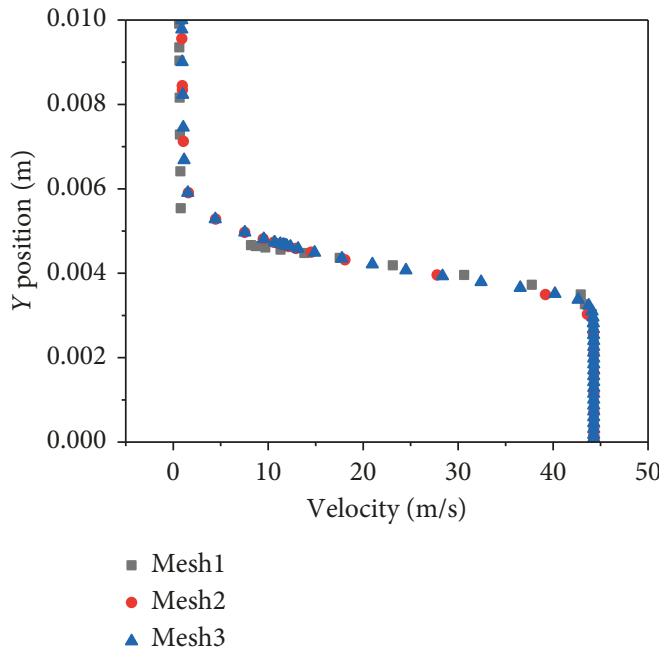

(a)

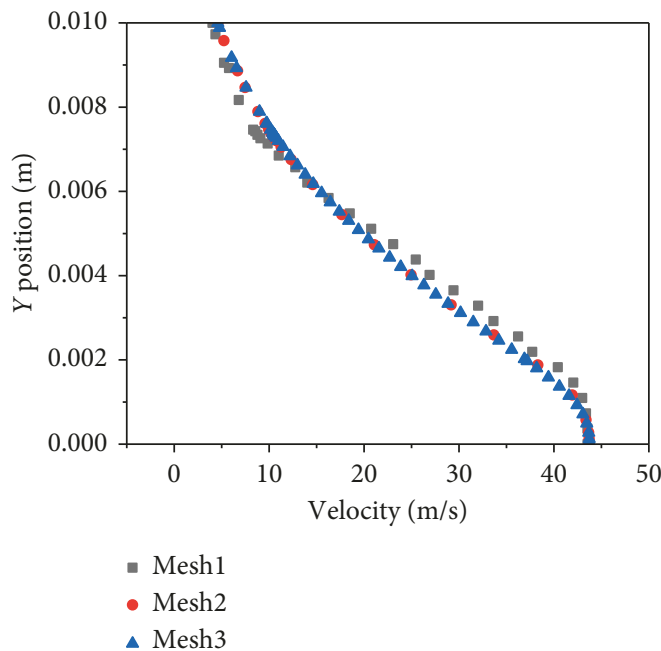

(c)

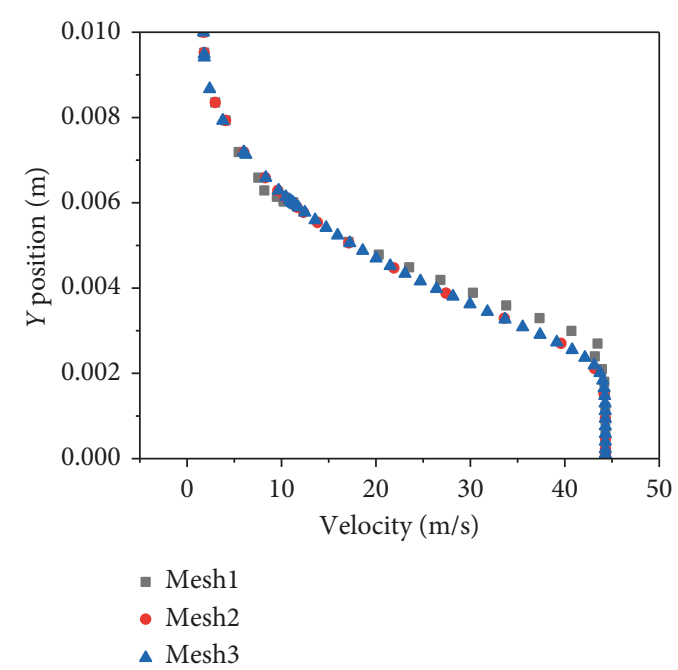

(b)

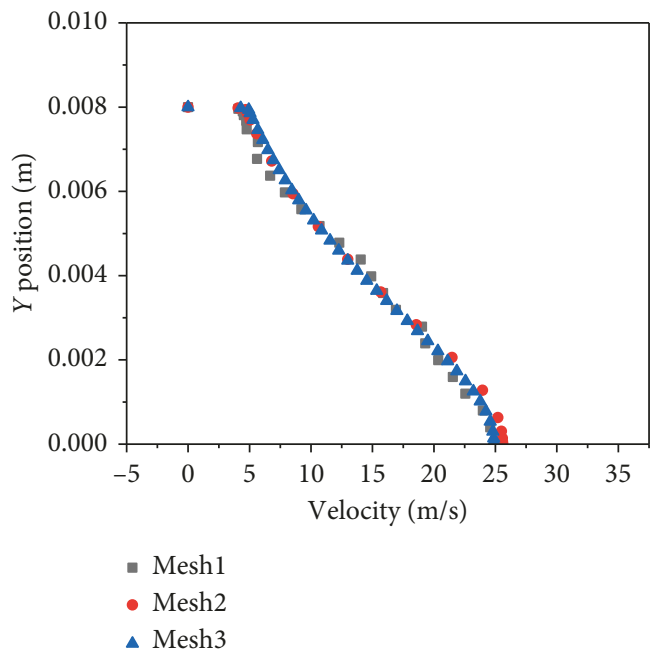

(d)

FIgURE 5: Distribution of monitoring line velocity. (a) Line 1. (b) Line 2. (c) Line 3. (d) Line 4.

fluctuation. From this perspective, in a cycle with pressure fluctuation, when pressure fluctuation of the gas nucleus occurs at the negative semiaxis, gas nucleus will expand. At this time, as the concentration of insoluble gas in gas nucleus decreases, insoluble gas of surrounding liquid will enter the gas nucleus. When pressure fluctuation occurs at the positive semiaxis, the gas nucleus will narrow. At this time, the concentration of insoluble gas in gas nucleus will increase, and insoluble gas will enter surrounding liquid. As the superficial area of the gas nucleus at the negative semiaxis is greater than that at the positive semiaxis, insoluble gas in the gas nucleus increases in this cycle with pressure fluctuation as a whole. Next, when the gas nucleus expands, the diffusion boundary layer of the gas nucleus will become thinner, which will increase the diffusion velocity of insoluble gas.

3.2.2. Pressure Management Area of Cavitating Jet Nozzle. Cavitation occurs under low pressure, so pressure distribution directly affects cavitation. Cavitation occurs after a period of time but does not occur suddenly. That is to say, the gas nucleus will expand to a critical radius after certain period in a low-pressure area before cavitation and cavitation will occur only when the flow field has certain lowpressure areas. Only when total pressure (sum of timeaverage pressure and fluctuation pressure) at a point of the flow field is lower than critical pressure of fluid, cavitation will occur (as shown in Figure 10). When water pressure periodically fluctuates, the gas nucleus will expand. This expansion mechanism is related to nonlinear effects within gas nucleus radius caused by periodic fluctuation. Pressure gradient has different influences on cavitation, fluctuation pressure, and development of boundary layer. Under large negative gradient, the development of boundary layer will be restrained and the pressure will decrease. In this case, cavitation will easily occur.

3.2.3. Analysis on Cavitation Status in Cavitating Jet Nozzle. Figure 11 shows different cavitation bubble status in the comparison between numerical simulation and experiment. 


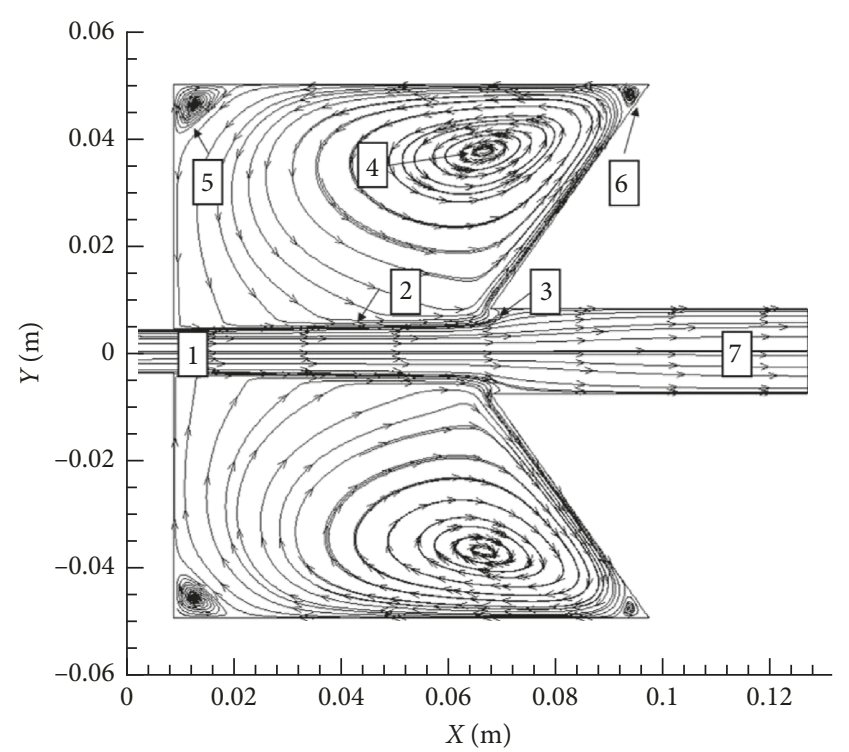

FIgURE 6: Time-averaged vorticity partitioning in cavitation jet nozzle.

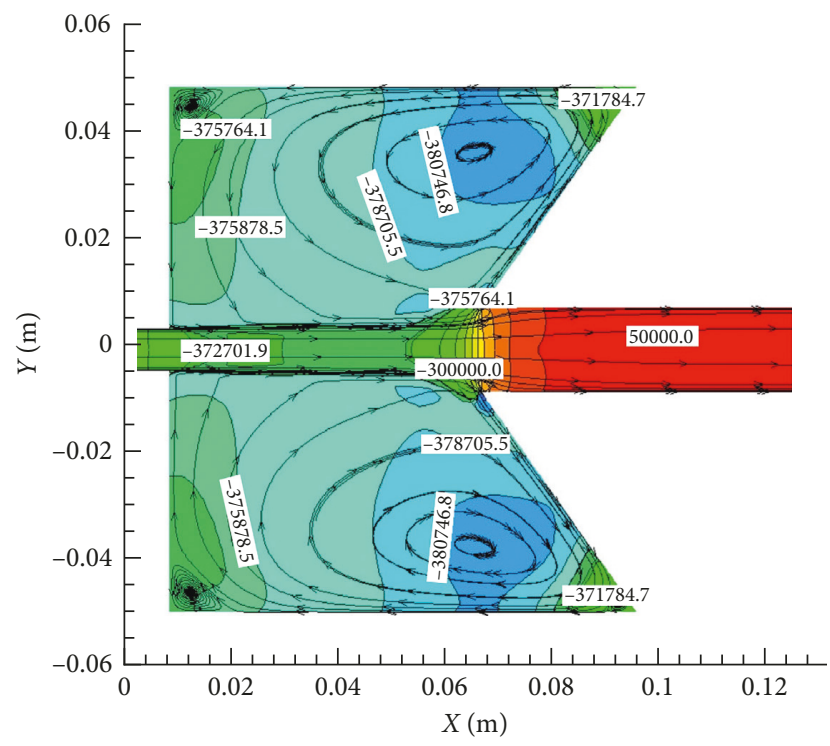

FIGURE 7: Time-averaged pressure nephogram before cavitation.

A change period diagram of one cavitation bubble was selected for analysis. The cavitation number was calculated at different periods. If $\delta=1.125$, it indicated that a gas nucleus appeared in the inlet of the upstream chamber and a single bubble rapidly changed at random. Compared with $\delta=1.094$ and $\delta=0.826$, the gas nucleus moved forward and the bubbles increased. The pressure management areas at this timeare shown in Figure 10. As can be seen from Figure 10, low-pressure gas existed in the center of the gas nucleus. At this time, the internal gas pressure was as low as $2000 \mathrm{~Pa}$, and the bubbles stayed away from the wall. As for two adjacent bubbles, a larger bubble acted as a wall and a small bubble would form jet for collapse of the large bubble. The existence time of the small bubble was directly proportional to the radius ratio $R 1 / R$ of these two bubbles. If the bubbles with same size collapsed at the same time, these bubbles would point at the middle jet. This period was an earlier stage of film-like cavitation. If $\delta=0.759$, exfoliated bubble cloud would appear, and this period was called as cloud cavitation period. Lots of small bubbles appeared around the gas-phase film and were aggregated into bubble cloud. Movements of bubble cloud were very complex and dramatic, and internal structure was changing. Formation, integration, breakdown, and collapse constantly occurred, and water and gas inversion and transduction were dramatic.

Figure 12 shows cross-sectional velocity distribution of exit longitudinal section. Information regarding the velocity at the exit was intercepted at different times. Before $\delta=1.094$, the boundary velocity was basically consistent and very gentle and high-velocity jet did not flow into the exit. Or, it could be interpreted as follows: flow in the chamber was in a laminar state at low initial velocity; after the velocity gradually increased to the turning point, the flow was in a high-velocity turbulent state. The downstream outlet pipe was thicker than the upstream pipe. According to an analysis based on Figure 13, jet flowed out from the axis and cavitation bubbles or cavitation nucleuses were formed at the up and down sides. Some cavitation nucleuses collapsed, which led to that some water flowed back and other water flowed with the large velocity gradient (i.e., high velocity at the center but low velocity at both sides) after high-velocity shearing.

3.2.4. Analysis on Pressure and Velocity at Nozzle Exit. The valve opening was further increased if cavitation bubbles did not appear. When vibration noises started to occur in the pipeline and gas nucleuses started to appear in the chamber, the outlet velocity and the pressure during the process were recorded by a pressure transmitter and an electromagnetic flowmeter. The relationship between the velocity and the time is shown in Figure 14 while the variation rule of the pressure over time is given in Figure 15. It could be seen that the cavitation velocity and pressure fluctuation were obviously higher than those without cavitation and the peak value of pressure fluctuation was up to 120 bar. As upstream flow increased to $Q=2 \mathrm{~m}^{3} / \mathrm{h}$, the velocity and peak pressure were very small. When the system was at an earlier cavitation stage, a little water was cavitated or no cavitation occurred and the boiling point was low. As the flow increased to $Q=3.6 \mathrm{~m}^{3} / \mathrm{h}$, the peak continuously increased. When $Q=7.2 \mathrm{~m}^{3} / \mathrm{h}$, the peak decreased. At this time, an optimum range of operating parameters could be obtained. The working range could be roughly divided into 3 areas (front, middle, and rear). The average of peak fluctuations in the front and rear areas was smaller than that in the middle area. At a high flow rate, large upstream and downstream oil pressure differences led to over high linear velocity on the center axis. Less cavitation bubbles and gas nucleuses were formed and were carried to the downstream exit, which would lead to that cavitation and its effects were affected.

Figure 16 shows the relationship between pressure pulsation and time in different flows. In order to analyze the cavitation speed, the whole flow was divided into 6 parts. By 


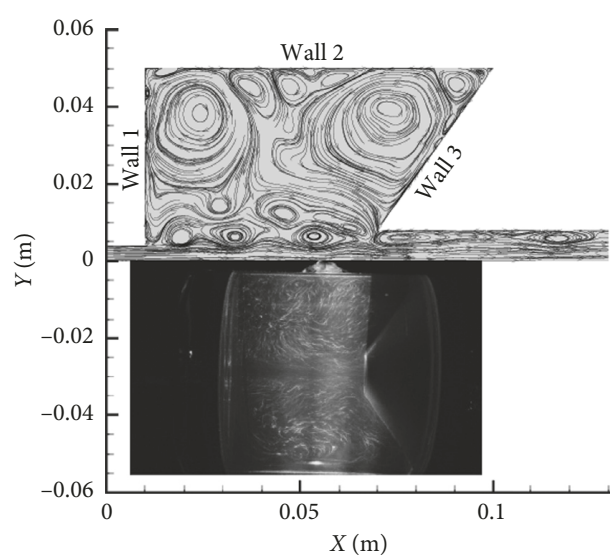

(a)

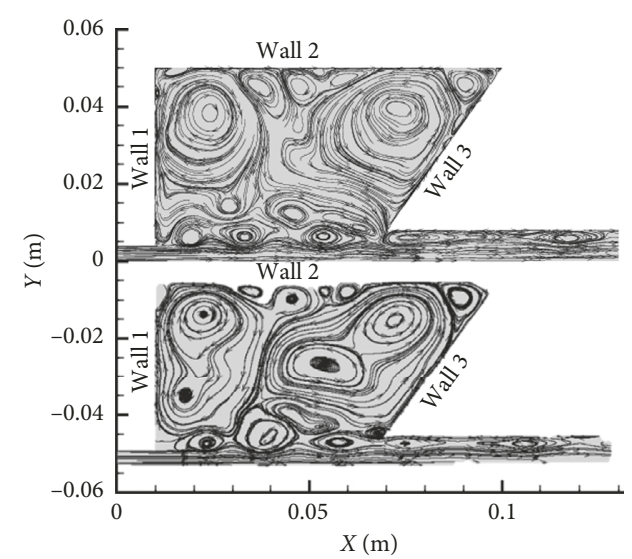

(b)

FIGURE 8: Instantaneous vorticity partitioning in cavitation jet nozzle. (a) Comparison diagram for numerical simulation and experiment. (b) Distribution of instantaneous vortex structure.

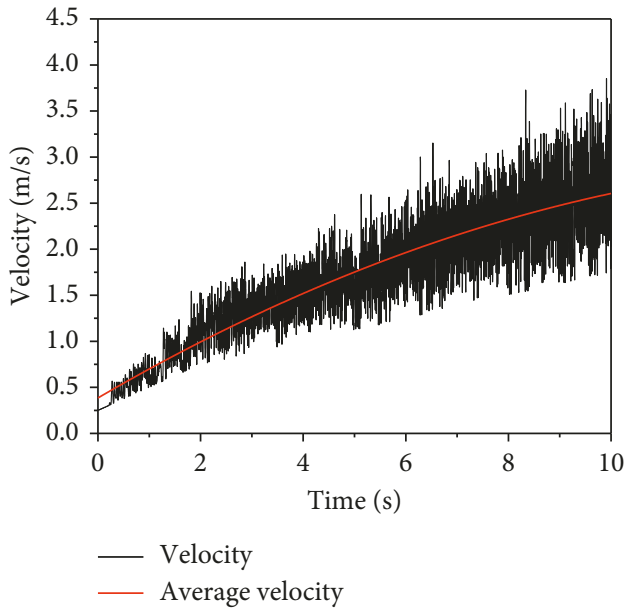

(a)

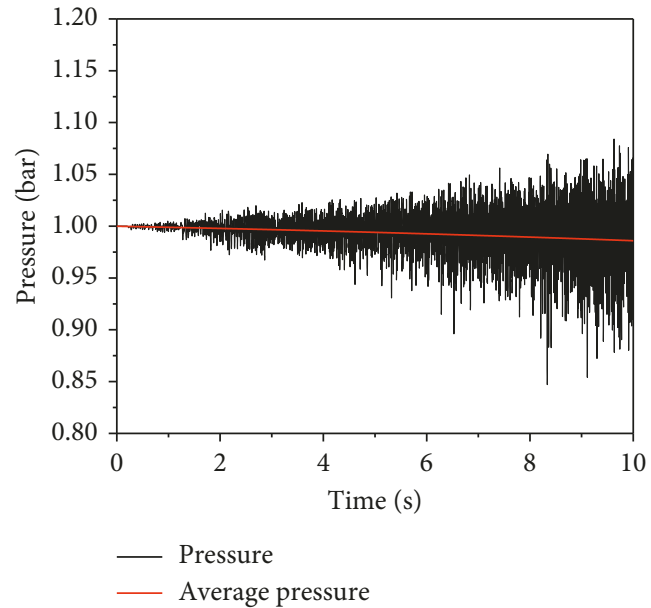

(b)

Figure 9: Curve for relationship between monitoring parameters and time. (a) Relationship between velocity and time. (b) Relationship between pressure and time.

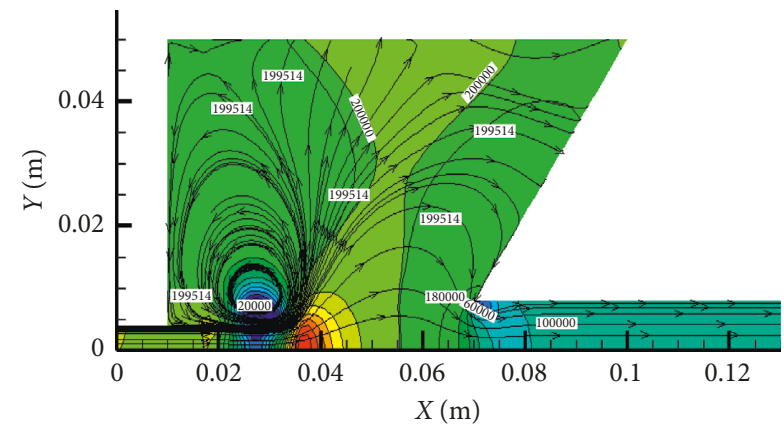

FIgURE 10: Cavitation pressure.

comparing peak value of pressure fluctuation and velocity amplitude at different flow rates, the peak ( $>5$ bar) most frequently appeared at $Q=7.2 \mathrm{~m}^{3} / \mathrm{h}$. Compared with $Q=4.5 \mathrm{~m}^{3} / \mathrm{h}$ and $Q=5.4 \mathrm{~m}^{3} / \mathrm{h}$, the peak value appeared for more than 11 times and the fluctuation amplitude also increased to a certain extent, presenting an overall increasing trend. At this time, cavitation bubbles increased. Compared with $Q=6.3 \mathrm{~m}^{3} / \mathrm{h}$ and $Q=5.4 \mathrm{~m}^{3} / \mathrm{h}$, the frequency of the appearance of the peak value and fluctuation amplitude was basically consistent. When $Q=7.2 \mathrm{~m}^{3} / \mathrm{h}$, a large number of extremely high peak values appeared, which conformed to the characteristics of cavitation stress concentration and large local pressure. At this time, the cavitation effect reached the optimum and the peak value appeared for up to 86 times.

Figure 17 shows the relationship between velocity and time in different flows. We would continue to discuss the velocity amplitude, establish upper and lower velocity envelops, and calculate the area based on envelop integral. As the time was selected to be $1 \mathrm{~s}$, the integral based area was approximately equal to velocity fluctuation amplitude. Through comparison, it was found that the velocity fluctuation amplitude was basically equivalent at $Q=7.2 \mathrm{~m}^{3} / \mathrm{h}$, $8.1 \mathrm{~m}^{3} / \mathrm{h}$, and $9.0 \mathrm{~m}^{3} / \mathrm{h}$. When the flow rate was $7.2 \mathrm{~m}^{3} / \mathrm{h}$, the 


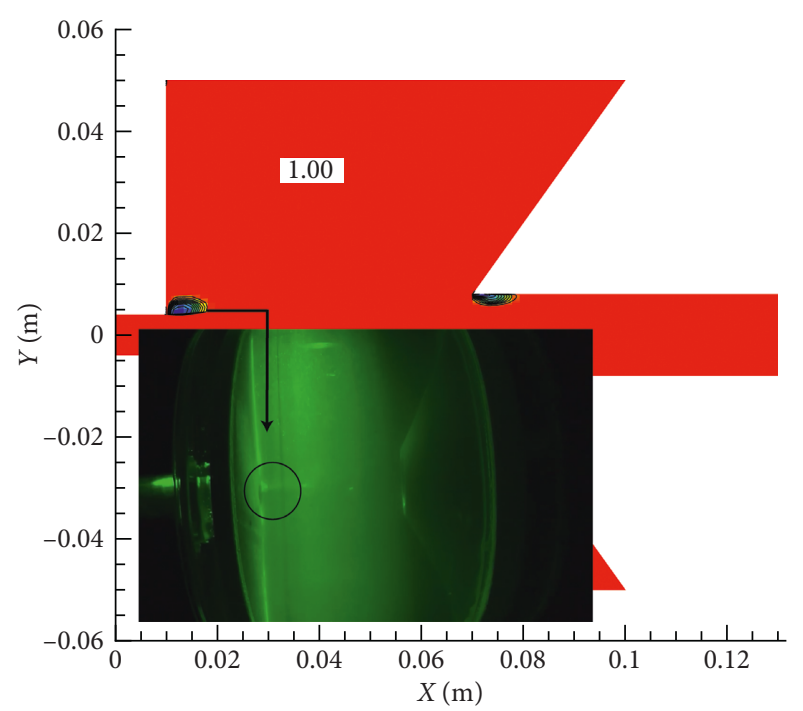

Phase 2:

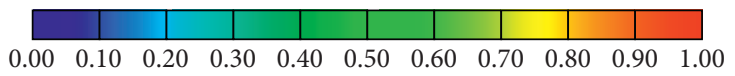

(a)

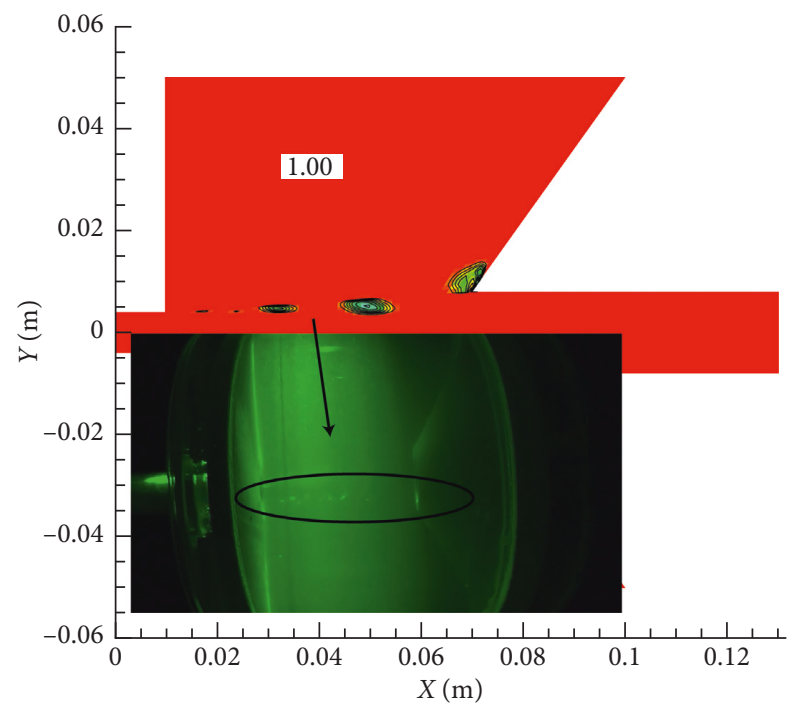

Phase 2:

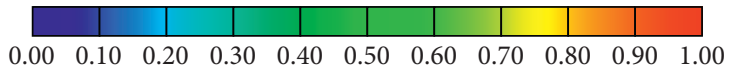

(c)

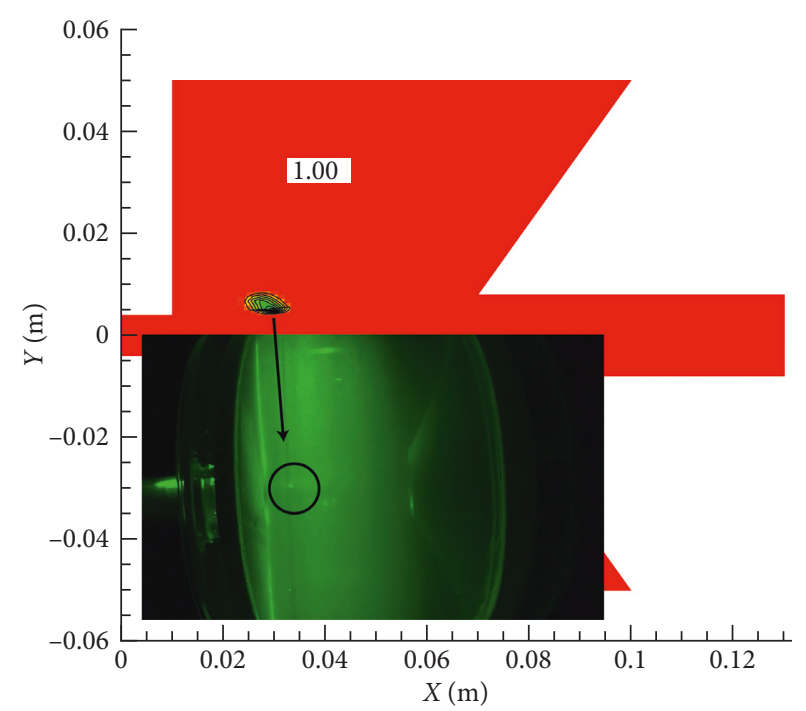

Phase 2:

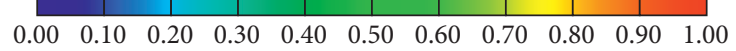

(b)

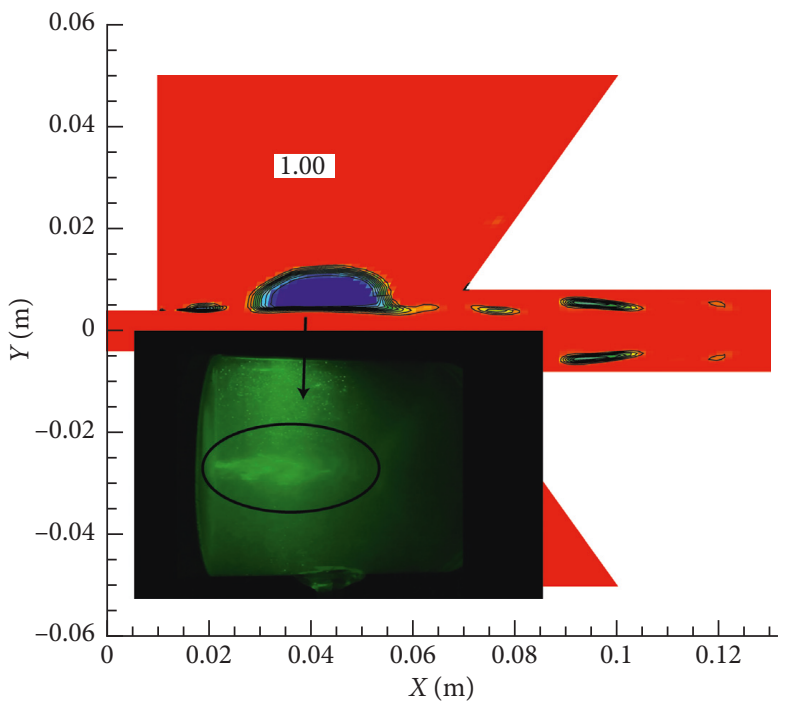

Phase 2:

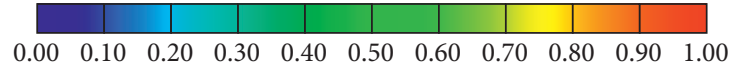

(d)

Figure 11: Cavitation status at different cavitation numbers. (a) $\delta=1.125$. (b) $\delta=1.094$. (c) $\delta=0.826$. (d) $\delta=0.759$.

amplitude was slightly higher. When $Q=4.5 \mathrm{~m}^{3} / \mathrm{h}$, $Q=5.4 \mathrm{~m}^{3} / \mathrm{h}$, and $Q=6.3 \mathrm{~m}^{3} / \mathrm{h}$, the velocity fluctuation amplitude increased from $6.69 \mathrm{~m}$ to $17.25 \mathrm{~m}$ and then became gentle or slightly decreased as the flow increased. The velocity fluctuation amplitude corresponded to pressure fluctuation frequency.

3.3. Analysis on Erosion Effects. In order to determine the correctness of analysis and validate cleaning effects of the cavitating jet nozzle at different flow rates, oxidation film on the surface of aluminum plate was flushed. During the experiment, we prepared a piece of $50 \times 50 \mathrm{~cm}$ square aluminum plate and flushed the fixed area of the aluminum plate for $90 \mathrm{~s}$ at different flow rates. After the experiment, we observed the erosion conditions of 6 areas to evaluate cleaning effects. The experiment results indicated that the effects reached the best when $Q=7.2 \mathrm{~m}^{3} / \mathrm{h}$ as shown in Figure 18.

The nozzle erosion results as shown in Figure 18 showed that the outside diameter and inside diameter of the erosion circle changed with the pressure and the area ratio of the actual erosion area to the entire erosion circle reflected the erosion efficiency. In Figure 18, the outside diameter and 


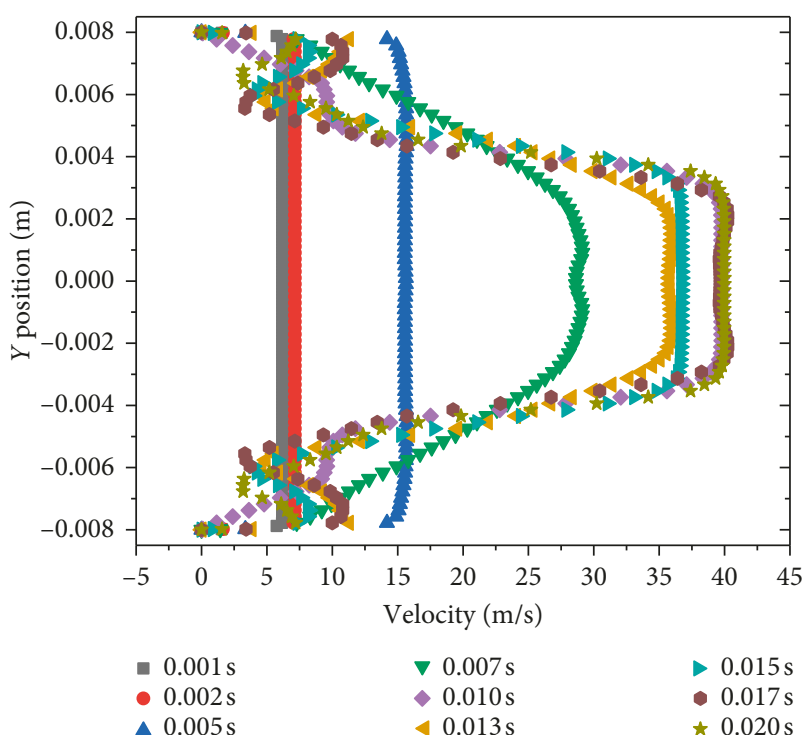

Figure 12: Exit velocity at different times.

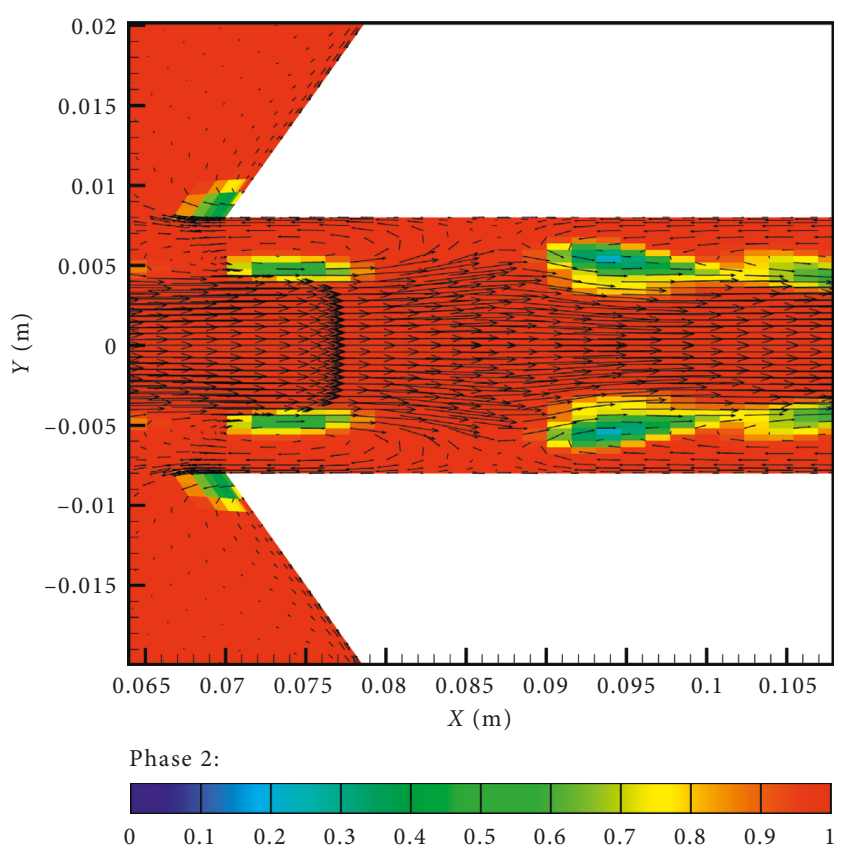

FIgURE 13: Vector diagram of downstream nozzle outlet.

inside diameter of the erosion circle were measured and the proportion of the erosion area was calculated. Figure 19 showed that the outside diameter of the erosion circle had little change at different flow rates. During the experiment, flow rates were selected in the normal working area, and cavitation and aluminum plate erosion occurred at the edges of the nozzle outlet. The flow rate increased, and the erosion efficiency increased with the pressure, reached the maximum $\left(Q=7.2 \mathrm{~m}^{3} / \mathrm{h}\right)$ and then decreased. It could be seen that, at a low flow rate, the eroded inner and outer circles were large and cleaning effects were not concentrated; at a high flow rate, the eroded inner circle was small, and erosion was relatively concentrated, with better effects than those at the

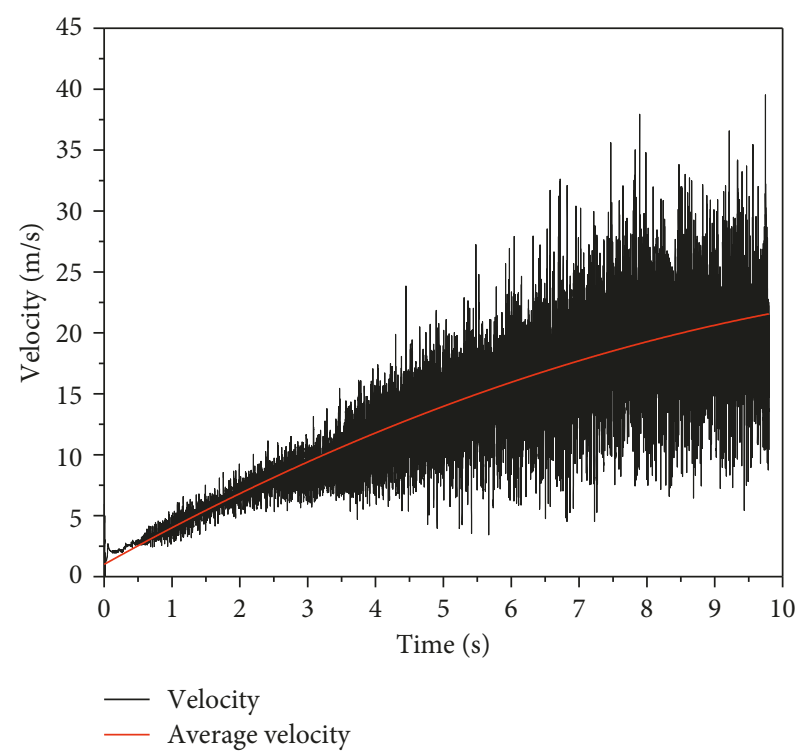

FIGURE 14: Relationship between velocity and time.

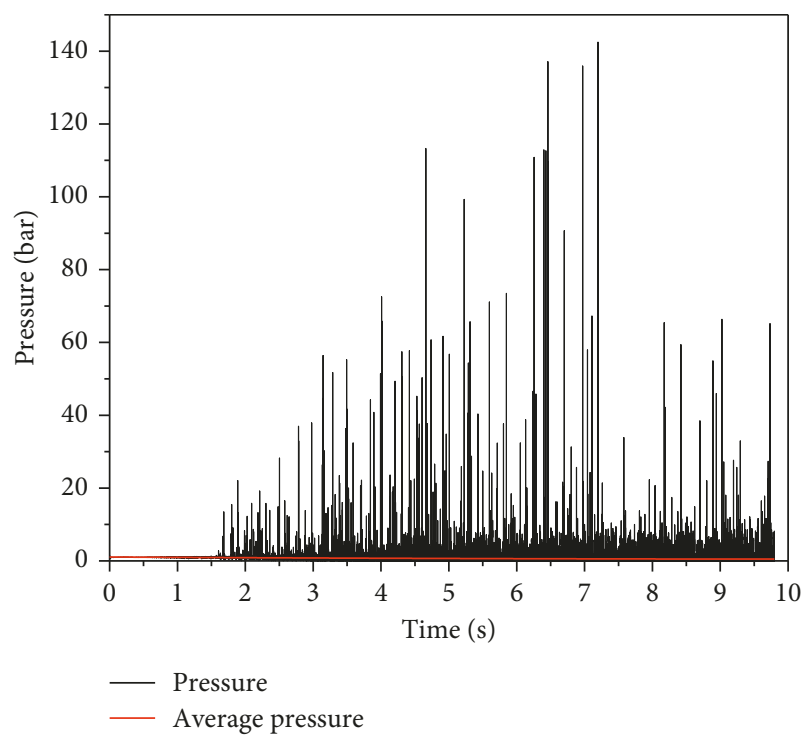

FIGURE 15: Relationship between pressure and time.

low flow rate. However, there was still a certain gap compared with the optimal working point.

\section{Conclusions}

Through analysis on pressure fluctuation frequency, velocity fluctuation amplitude, vortex structure change, and cavitation number of the cavitation jet nozzle at different flow rates, following conclusions can be obtained:

(1) At a flow rate lower than $2.7 \mathrm{~m}^{3} / \mathrm{h}$, no cavitation occurred in the nozzle. However, due to pressure and velocity fluctuation caused by resonance and periodic change of large vortex structure, the velocity fluctuation amplitude before cavitation was about $17 \%$ of that after cavitation, and the flushing effects 


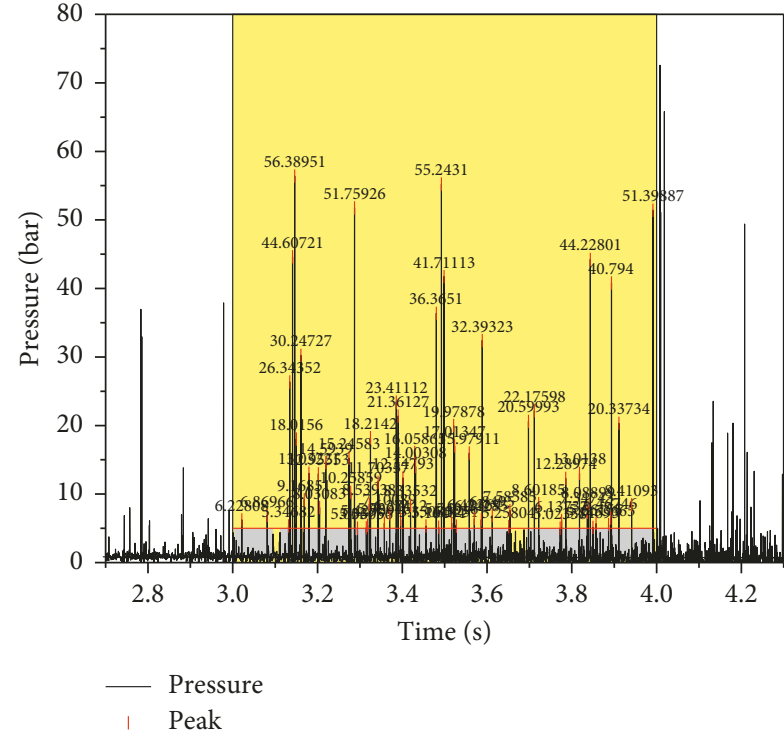

(a)

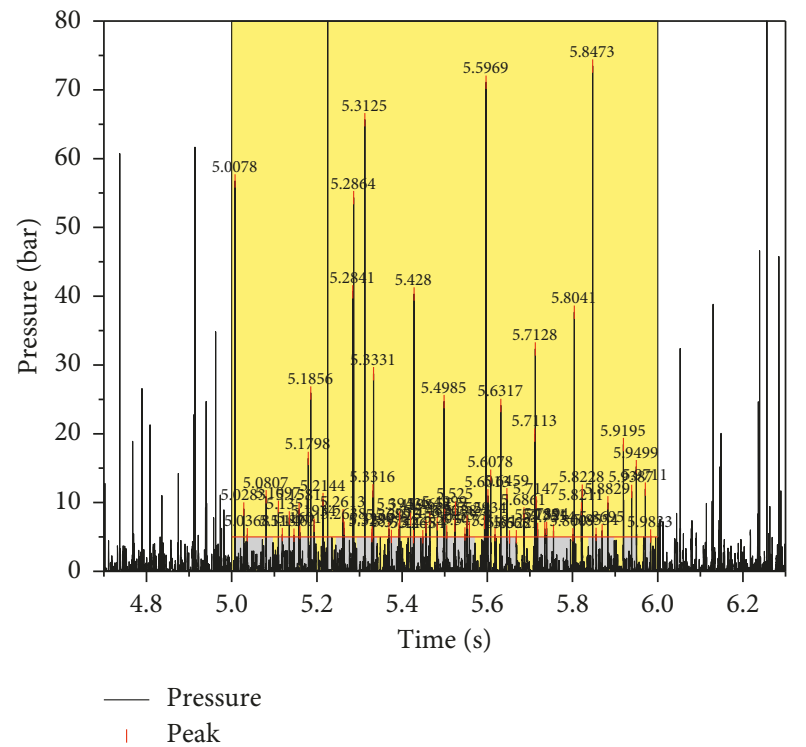

(c)

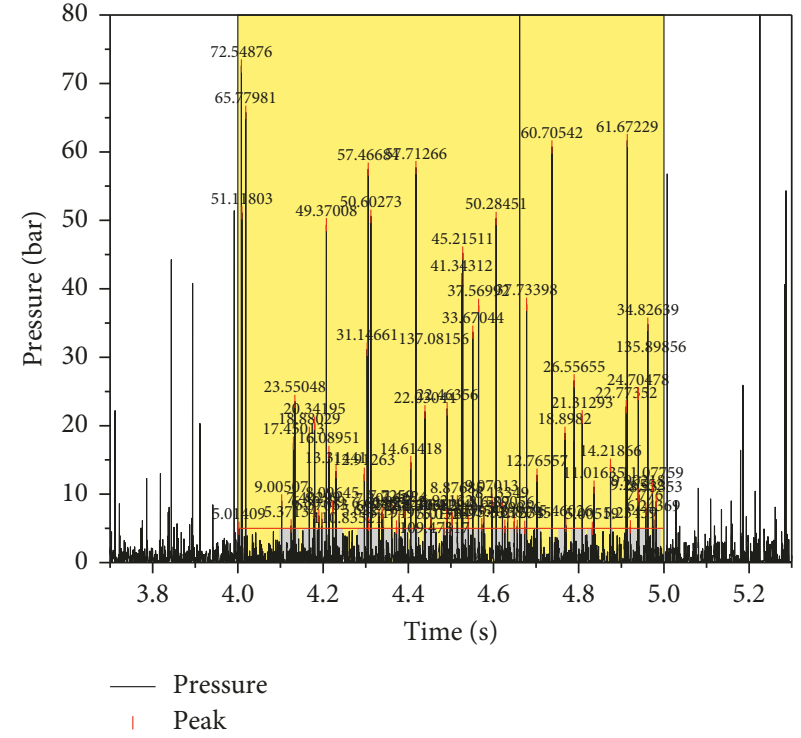

(b)

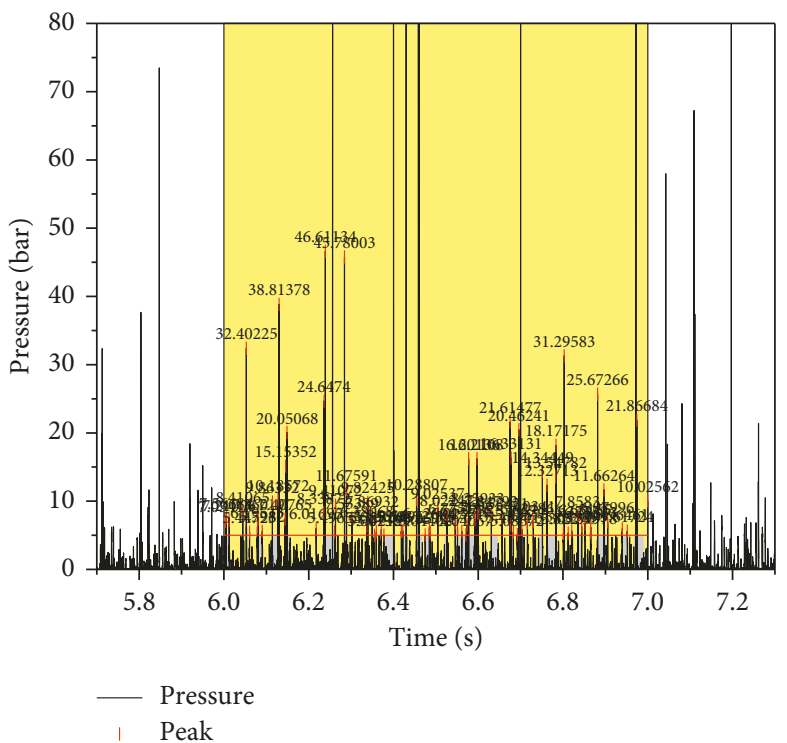

(d)

Figure 16: Continued. 

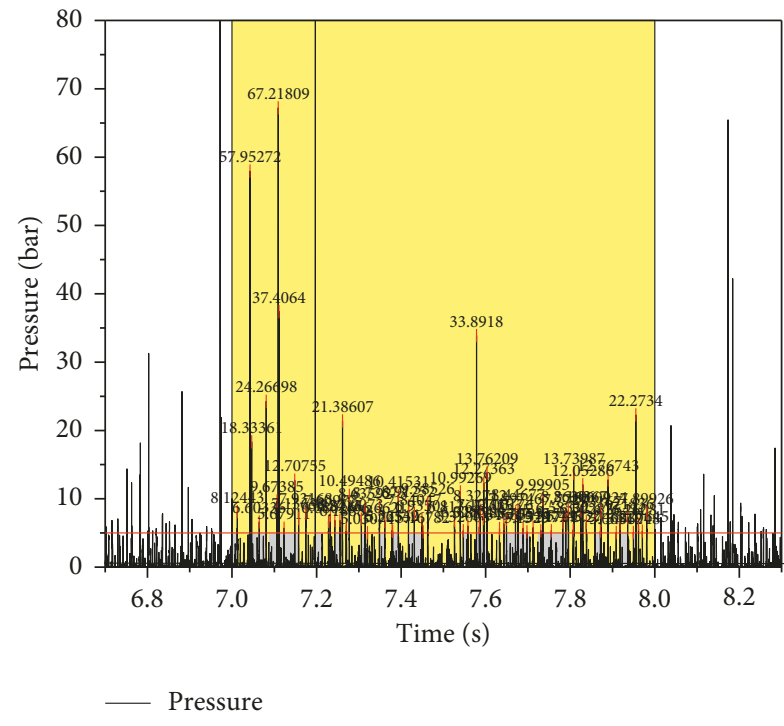

Peak

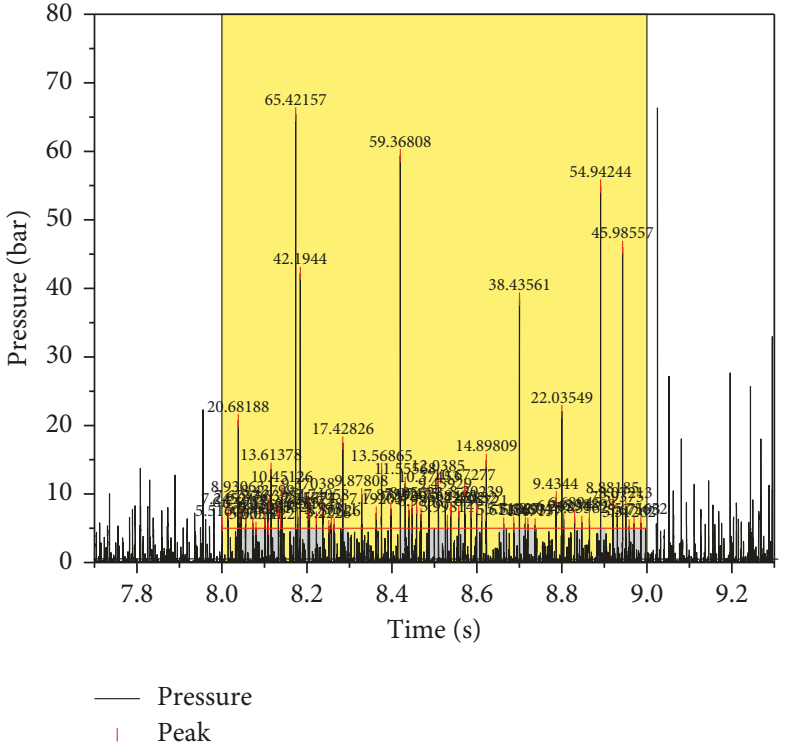

(f)

FiguRE 16: Curve for relationship between pressure fluctuation and time at different flow rates. (a) Found 65 peaks, $Q=4.5 \mathrm{~m}^{3} / \mathrm{h}$. (b) Found 76 peaks, $Q=5.4 \mathrm{~m}^{3} / \mathrm{h}$. (c) Found 73 peaks, $Q=6.3 \mathrm{~m}^{3} / \mathrm{h}$. (d) Found 86 peaks, $Q=7.2 \mathrm{~m}^{3} / \mathrm{h}$. (e) Found 74 peaks, $Q=8.1 \mathrm{~m}^{3} / \mathrm{h}$. (f) Found 70 peaks, $Q=9.0 \mathrm{~m}^{3} / \mathrm{h}$.

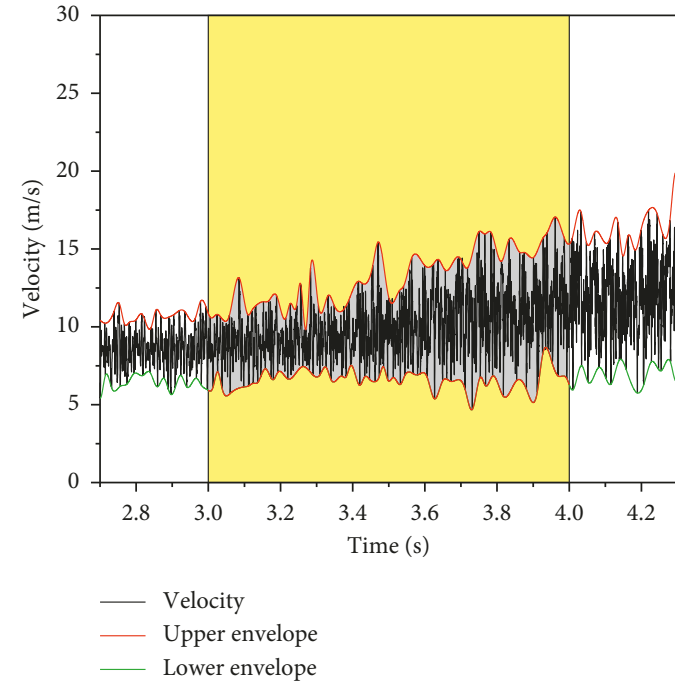

(a)

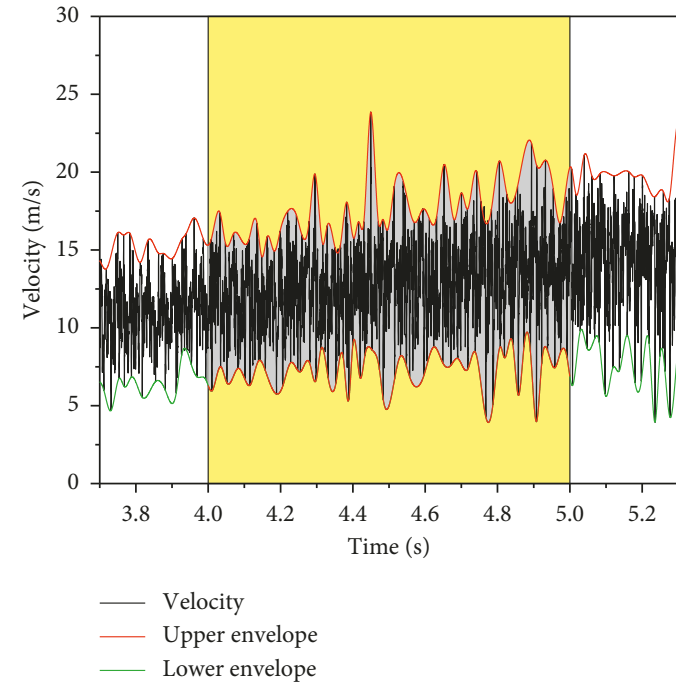

(b)

FIgURE 17: Continued. 


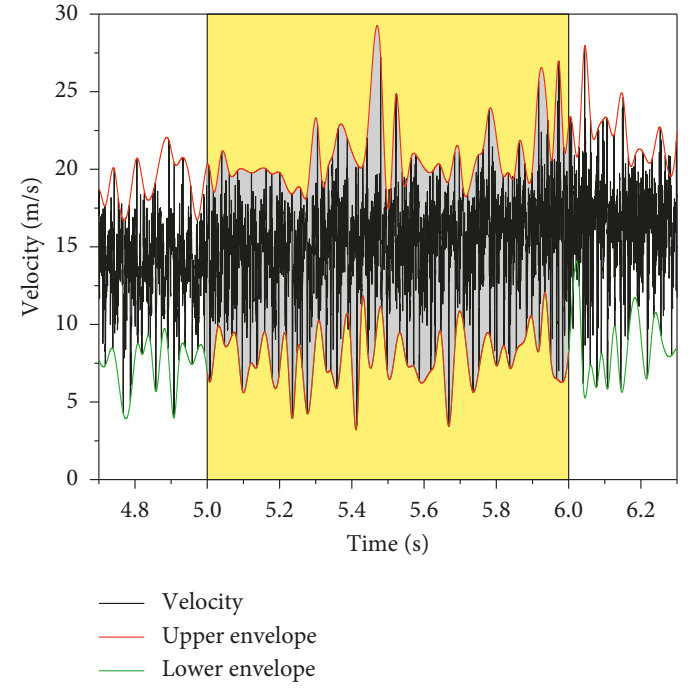

(c)

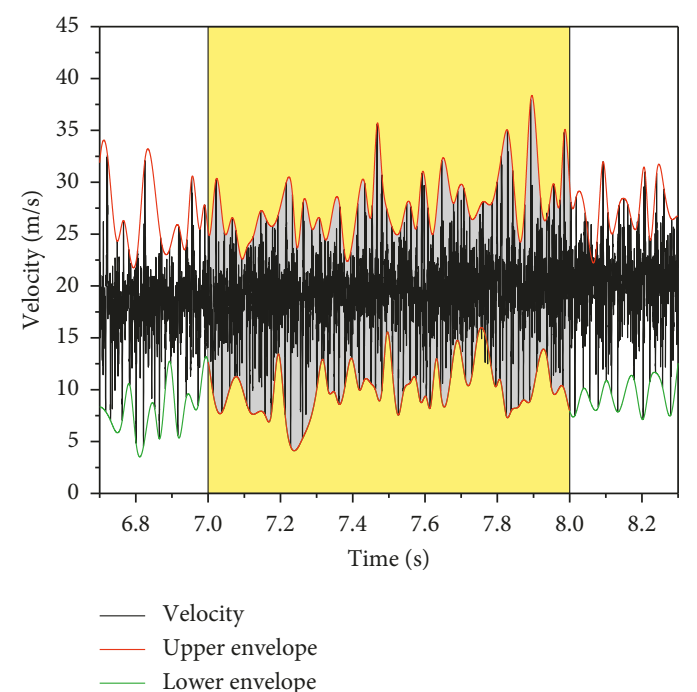

(e)

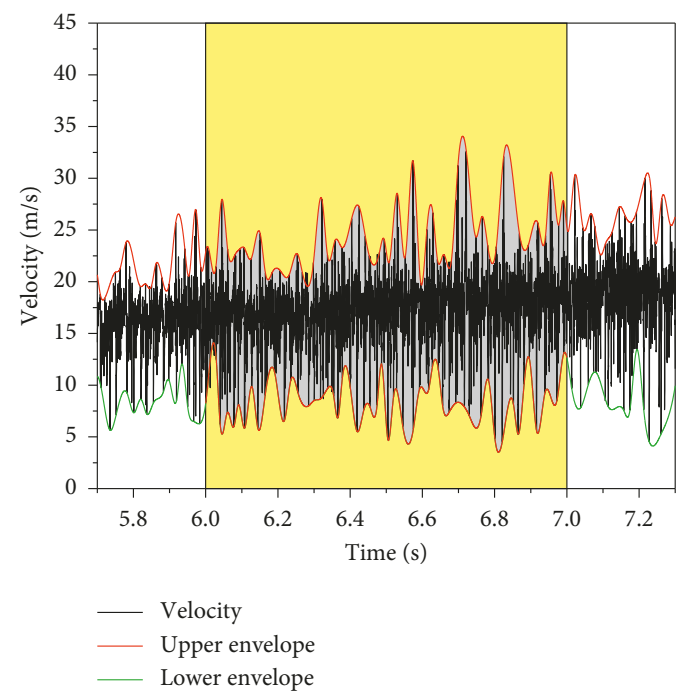

(d)

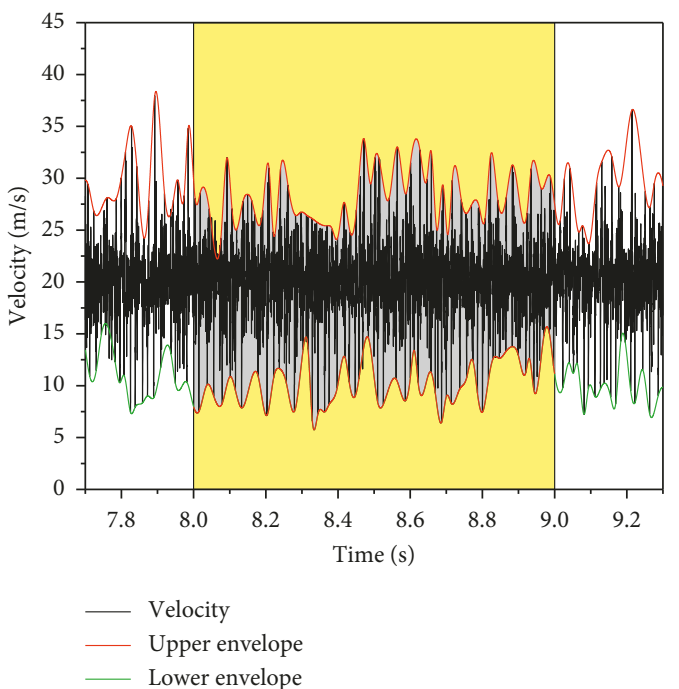

(f)

Figure 17: Curve for relationship between velocity and time at different flow rates. (a) Area $=6.69, Q=4.5 \mathrm{~m}^{3} / \mathrm{h}$. (b) Area $=10.50$, $Q=5.4 \mathrm{~m}^{3} / \mathrm{h}$. (c) Area $=13.05, Q=6.3 \mathrm{~m}^{3} / \mathrm{h}$. (d) Area $=17.25, Q=7.2 \mathrm{~m}^{3} / \mathrm{h}$. (e) Area $=16.88, Q=8.1 \mathrm{~m}^{3} / \mathrm{h}$. (f) Area $=17.06, Q=9.0 \mathrm{~m}^{3} / \mathrm{h}$.

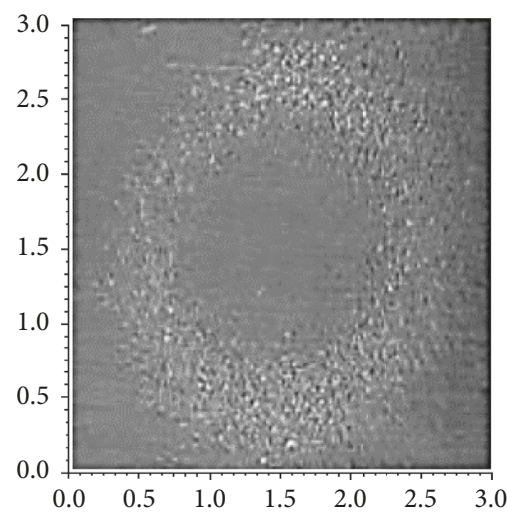

(a)

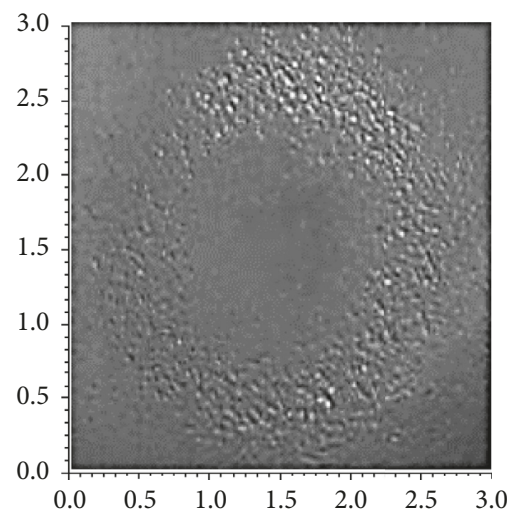

(b)

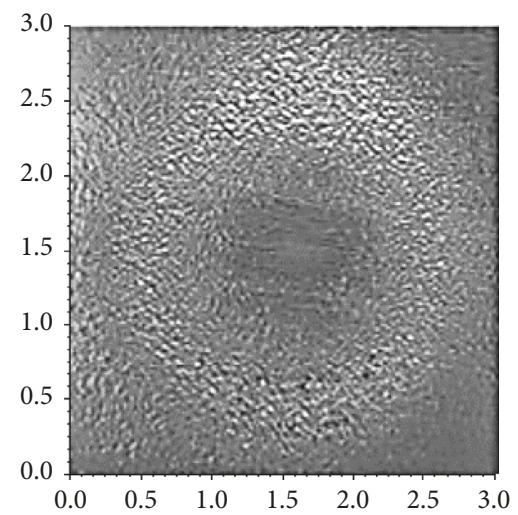

(c)

Figure 18: Continued. 


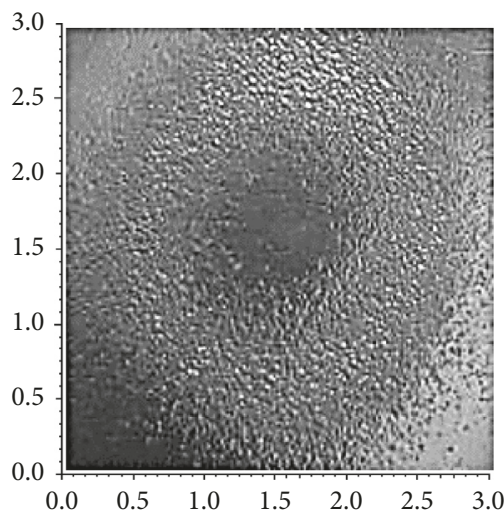

(d)

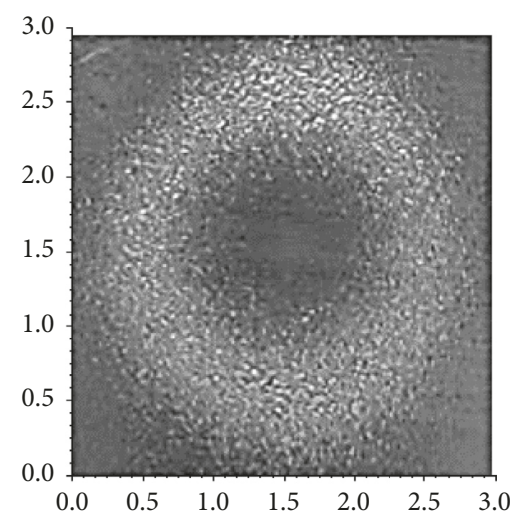

(e)

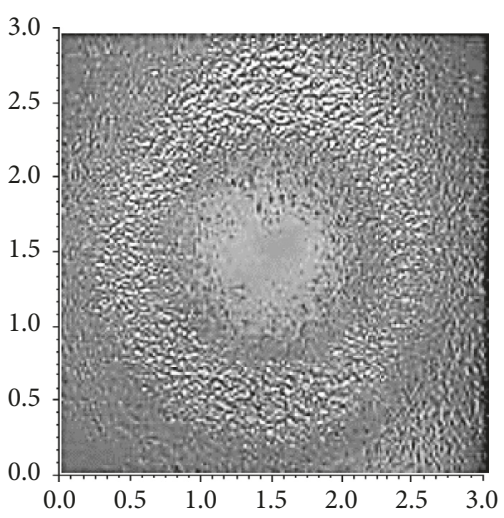

(f)

Figure 18: Erosion effects at different flow rates. (a) $Q=4.5 \mathrm{~m}^{3} / \mathrm{h}$. (b) $Q=5.4 \mathrm{~m}^{3} / \mathrm{h}$. (c) $Q=6.3 \mathrm{~m}^{3} / \mathrm{h}$. (d) $Q=7.2 \mathrm{~m}^{3} / \mathrm{h}$. (e) $Q=8.1 \mathrm{~m}^{3} / \mathrm{h}$. (f) $Q=9.0 \mathrm{~m}^{3} / \mathrm{h}$.

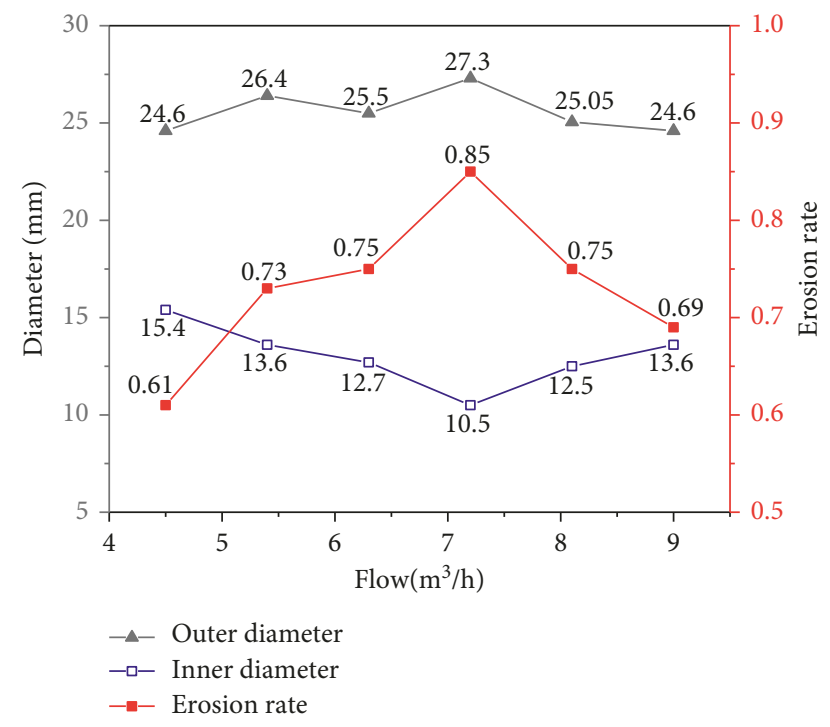

Figure 19: Erosion effects at different flow rates.

were far below those in the chamber where cavitation occurred.

(2) When the flow rate increased to over $2.7 \mathrm{~m}^{3} / \mathrm{h}$, cavitation started to occur in the chamber. Through numerical simulation, pressure distribution in the chamber was analyzed and the positions and status of the cavitation bubbles were predicted. By comparing the results with experiment results, it indicated that less cavitation brought the best cavitation effects.

(3) The pressure fluctuation frequency and velocity fluctuation amplitude at different flow rates were analyzed through experiment, and statistics and integral analysis were given on experimental data. The velocity fluctuation amplitude was about $17.25 \mathrm{~m}$ at $Q=7.2 \mathrm{~m}^{3} / \mathrm{h}$ and was approximate to that at $Q=7.2 \mathrm{~m}^{3} / \mathrm{h}$ and $9.0 \mathrm{~m}^{3} / \mathrm{h}$. Pressure fluctuation occurred for 86 times (maximum) within $1 \mathrm{~s}$.

(4) In order to validate the above analysis results, experiments on erosion effects at 6 different flow rates were conducted. And, outside diameter, inside diameter, and erosion rate of the erosion circle were measured. Under experiment conditions, flow rate had little influence on outside diameter of the erosion circle. The erosion rate increased with the increase of the flow rate, reached the peak value at $Q=7.2 \mathrm{~m}^{3} / \mathrm{h}$, but slightly decreased subsequently.

\section{Data Availability}

The data used to support the findings of this study are available from the corresponding author upon request.

\section{Conflicts of Interest}

The authors declare they have no conflicts of interest.

\section{Authors' Contributions}

In this research activity, all authors were involved in the data collection and preprocessing phase, model construction, empirical research, result analysis and discussion, and manuscript preparation. All authors have approved the submitted manuscript.

\section{Acknowledgments}

This work was supported by the PetroChina Innovation Foundation (No. 2018D-5007-0206). The completion of this paper has been helped by many teachers and classmates. We would like to express our gratitude to them for their help and guidance.

\section{References}

[1] L. Y. Wang, X. Kang, and Y. Chen, "Numerical simulation of cavitation jet flow field generated by scaling nozzles," Journal of China University of Petroleum (Natural Science Edition), vol. 33, no. 6, pp. 57-60, 2009.

[2] K. W. Peng, S. Z. Tian, G. S. Li et al., "Natural cavitating jet kinetic characteristics of cavitation and the breaking strength 
influencing factors," Journal of Petroleum Exploration and Development, vol. 45, no. 2, pp. 326-332, 2018.

[3] V. E. Johnson and A. F. Conn, "The fluid dynamics of submerged water jets," in Proceedings of the 5th International Symposium on Jet Cutting Technology, pp. 201-209, HBRA, Hannover, Germany, 1976.

[4] A. Kubota, H. Kato, and H. Yamaguchi, "A new modeling of cavitating flows: a numerical study of unsteady cavitation on a hydrofoil section," Journal of Fluid Mechanics, vol. 240, no. 1, pp. 59-96, 1992.

[5] M. Geveci, P. Oshkai, D. Rockwell, J.-C. Lin, and M. Pollack, "Imaging of the self-excited oscillation of flow past a cavity during generation of a flow tone," Journal of Fluids and Structures, vol. 18, no. 6, pp. 665-694, 2003.

[6] M. M. Vijay, C. Zou, S. G. Hu, J. Remisz, and S. Tavoularis, "A study of the practicality of cavitating water jets," in Proceedings of the Jet Cutting Technology, vol. 13, pp. 75-99, Springer, Dordrecht, Netherlands, 1992.

[7] Z. Wang and Z. Shen, "Numerical study on the influence of the throat structure of the organ nozzle on the flow field of the jet," Hydrodynamics Research and Progress, vol. 10, no. 5, pp. 474-478, 1995.

[8] S. Deng, Y. Shen, Z. Li et al., "Numerical simulation of cavitation jet nozzle flow field," Journal of Logistics Engineering College, vol. 24, no. 2, pp. 42-46, 2008.

[9] X. Ruliang, Studies on the technologies of jet cleaning, sludge forming and resourceful treatment for the industrial oil tank, Zhejiang University, Hangzhou, China, 2004.

[10] Y. L. Jiang, L. Wang, and R. Xu, "Cavition model for the lowpressure large-diameter self-excited pulse nozzle," Journal of Engineering Thermophysics, vol. 26, pp. 438-440, 2005.

[11] J. Li, R. Xu, and L. Wang, "Numerical simulation of mechanism of the self-excited pulse nozzle," Journal of Engineering Thermophysics, vol. 25, pp. 241-243, 2004.

[12] C. Gao, H. Chen, and T. Lei, "Research and progress of the self-excited oscillation pulsed jet," Journal of North China Institute of Water Conservancy and Hydroelectric Power, vol. 30, pp. 41-44, 2009.

[13] S. Chen, C. Li, and Y. Qu, "Research on the effect factors of the novel self-excited oscillation pulse jet," Journal of Shandong University (Engineering Science), vol. 36, pp. 12-15, 2006.

[14] Y. Qu, C. Li, and R. Su, "The numerical analysis on structural parameters of self-excited pulsed jet to the eddy ring," Journal of Shandong University (Engineering Science), vol. 36, pp. 17-21, 2006.

[15] Y. Qu, S. Chen, and C. Li, "The numerical simulation of liquid-vapor two phase flow in low pressure-high flow rate self-excited pulsed jet nozzle," Journal of Shandong University (Engineering Science), vol. 36, pp. 16-20, 2006.

[16] A. Powell, "On the edgetone," Journal of the Acoustical Society of America, vol. 33, no. 4, pp. 395-409, 1961.

[17] T. A. Wilson, G. S. Beavers, M. A. Decoster, D. K. Holger, and M. D. Regenfuss, "Experiments on the fluid mechanics of whistling," Journal of the Acoustical Society of America, vol. 50, no. 1B, pp. 366-372, 1971.

[18] S. C. Crow and F. H. Champagne, "Orderly structure in jet turbulence," Journal of Fluid Mechanics, vol. 48, no. 3, pp. 547-591, 1971.

[19] M. S. Howe, "On the Helmholtz resonator," Journal of Sound and Vibration, vol. 45, no. 3, pp. 427-440, 1976.

[20] R. Ma, P. E. Slaboch, and S. C. Morris, "Fluid mechanics of the flow-excited Helmholtz resonator," Journal of Fluid Mechanics, vol. 623, pp. 1-26, 2009.
[21] R. Corá, C. A. Martins, and P. T. Lacava, "Acoustic instabilities control using Helmholtz resonators," Applied Acoustics, vol. 77, pp. 1-10, 2014.

[22] D. Hu, X.-h. Li, C.-L. Tang, and Y. Kang, "Analytical and experimental investigations of the pulsed air-water jet," Journal of Fluids and Structures, vol. 54, pp. 88-102, 2015.

[23] C. L. Tang, D. Hu, and F. H. Zhang, "Study on the frequency characteristic of self-excited oscillation pulsed water jet," Advanced Materials Research, vol. 317-319, pp. 1456-1461, 2011.

[24] C.-T. Hsiao, G. L. Chahine, and H.-L. Liu, Scaling Effects on Bubble Dynamics in a Tip Vortex Flow: Prediction of Cavitation Inception and Noise, Report, Dynaflow Inc, Jessup, MD, USA, 2000. 


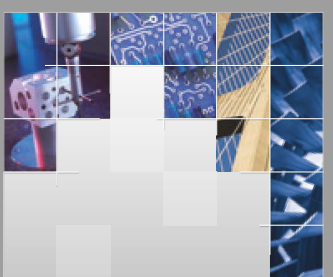

\section{Enfincering}
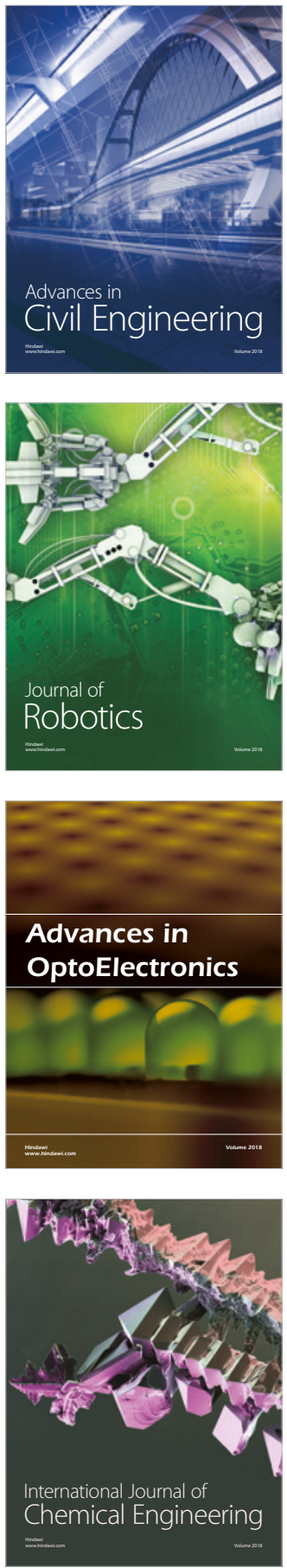

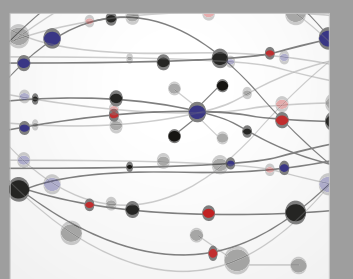

\section{Rotating \\ Machinery}

The Scientific World Journal

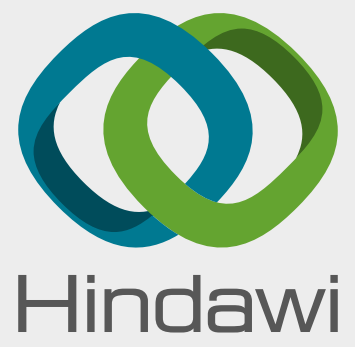

Submit your manuscripts at

www.hindawi.com
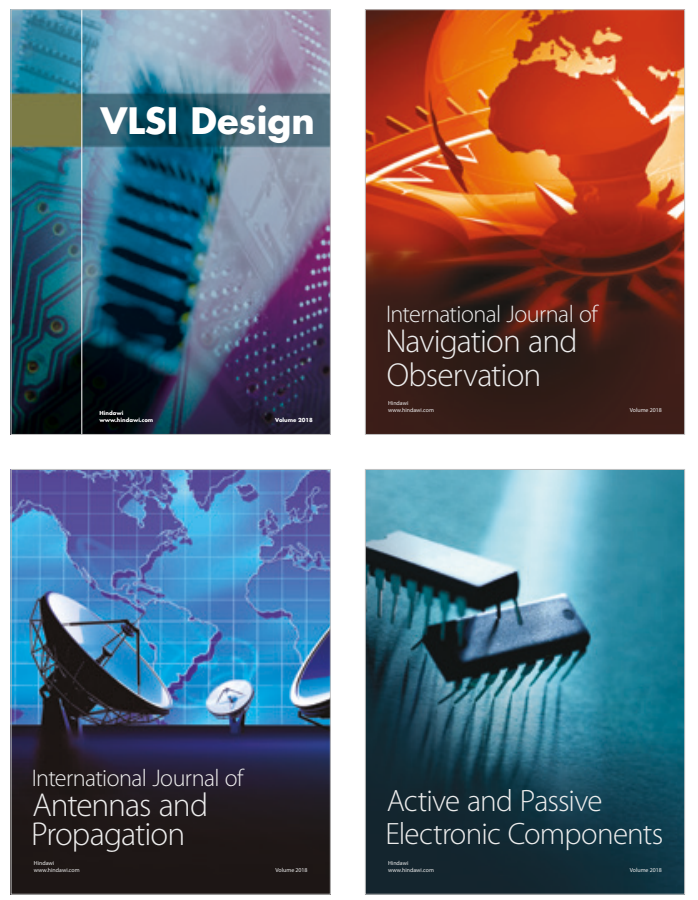
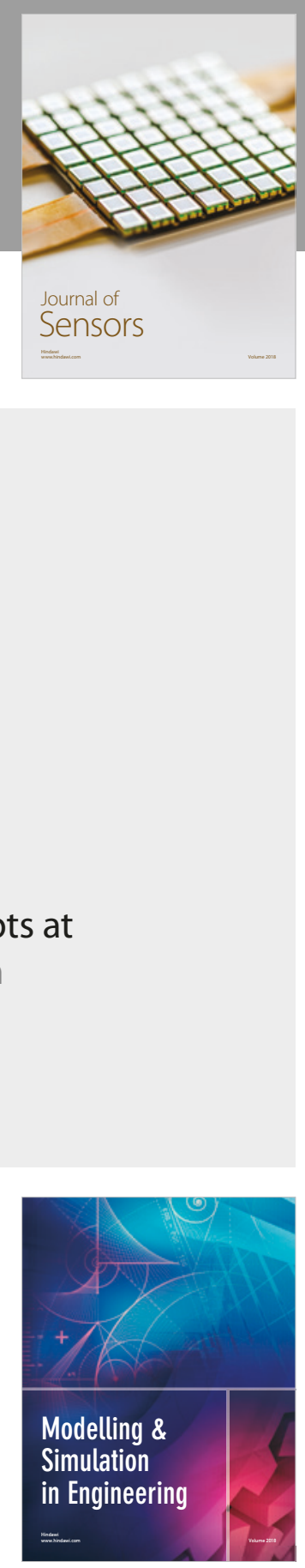

\section{Advances \\ Multimedia}
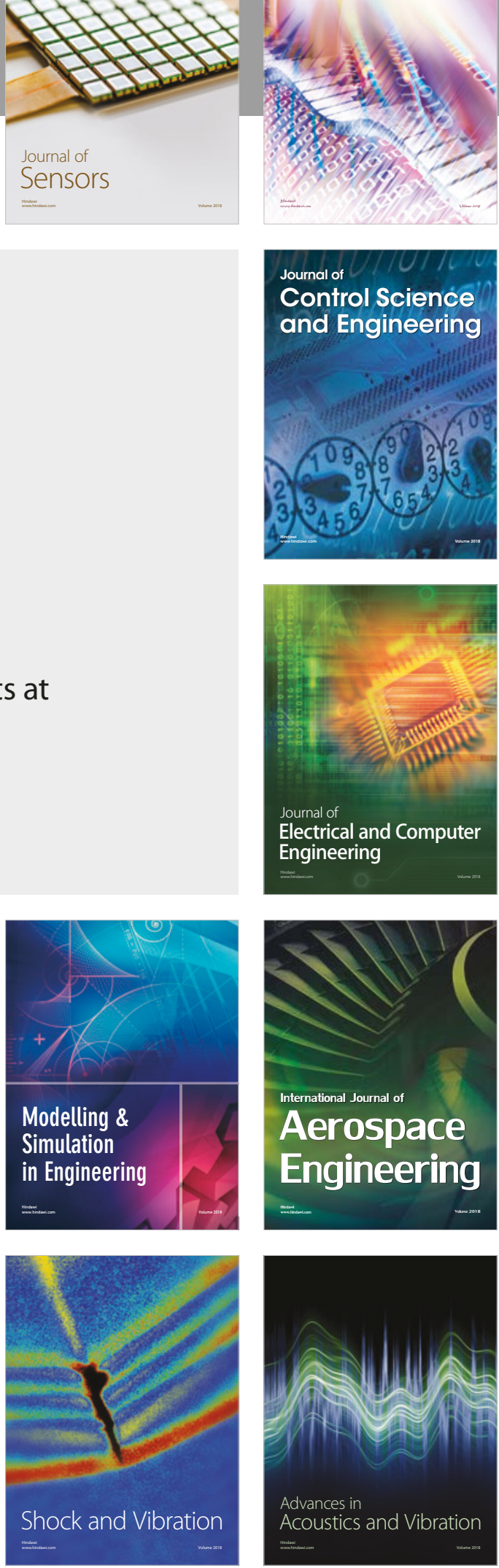